\title{
INVITED SURVEY PAPER Statistical Multipath Propagation Modeling for Broadband Wireless Systems
}

SUMMARY This paper surveys and introduces propagation studies and models that are expected to contribute to the development of broadband wireless systems. The survey focused on theory-based propagation models, experimental measurement data useful for modeling, and transmission characteristic evaluations using propagation models. The survey did not attempt to cover all papers in the research fields, but rather took key papers for various relevant subjects and described them in some detail. The basic characteristics of multipath propagation are summarized from the viewpoints of narrow-band (NB), wide-band (WB), and ultra wide-band (UWB). Recent studies on spatio-temporal propagation models and the relationship between models and systems are introduced. To clarify the relationship between OFDM, which is a representative of wideband data transmission schemes, and wave propagation factors, problems due to large delay spread and large Doppler spread are highlighted. Finally, studies on UWB propagation measurement and propagation models are introduced. key words: broadband wireless, multipath propagation, propagation model, UWB angular power profile

\section{Introduction}

In the design of wireless communication systems, wave propagation studies are very important. In particular, in broadband mobile communications for high-speed data transmission, propagation delay produces waveform distortion and generates transmission errors due to inter-symbol interference (ISI). Therefore, wideband propagation has been studied energetically from this point of view since the 1980s [1]-[3]. As represented by adaptive arrays and multiinput multi-output (MIMO) systems, high channel capacity transmissions using antenna array are becoming more and more attractive [4]-[7]. In this case, the angular distribution characteristic of the incoming wave is of great interest for wave propagation models. The problem of rapid phase variations due to Doppler spread had become insignificant for single-carrier wideband systems, but with the advent of orthogonal frequency-division multiplexing (OFDM) transmission for wireless mobile applications, this propagation problem has again become apparent. Currently, research and development are advancing for very large relative bandwidth systems, called ultra wide-band (UWB) systems. Various propagation studies for UWB are being conducted to develop propagation models those cannot be covered by extending conventional propagation models for narrow-band (NB) or wide-band (WB) systems [8]-[10].

This survey paper considers propagation studies and

Manuscript received November 14, 2006.

${ }^{\dagger}$ The author is with AWCC, Univ. Electro-Communications, Chofu-shi, 182-8585 Japan.

a)E-mail: karasawa@ee.uec.ac.jp

DOI: $10.1093 /$ ietcom/e90-b.3.468 propagation models that are likely to contribute to developing broadband wireless systems. It focuses on theoretical propagation models and experimental measurement data useful for modeling. On the other hand, empirical propagation models based on experimental data for propagation loss estimation methods [11], [12], and system-oriented propagation models for performance evaluation of specific systems [13], [14] are excluded from this survey. In addition, MIMO propagation channel models [15]-[18] have been omitted, despite growing expectations for their technologies, because of preventing from divergence of this survey.

This survey paper does not attempt a complete review of the subject fields, but rather presents key papers for various relevant topics and describes them in some detail. In other words, this paper is not intended to be a list of papers (like a catalog), but instead gives sufficient information about the papers so that the reader need not return to the originals for brief understanding. Section 2 summarizes the basic characteristics of multipath propagation from the viewpoint of NB, WB, and UWB. Section 3 introduces recent studies on spatio-temporal propagation models and the relationship between the models and systems. To identify the necessary condition of OFDM parameters and wave propagation parameters, the first half of Sect. 4 describes a mechanism where ODFM system design becomes difficult in future communication systems operated in high speed vehicles at higher frequency. The second half of Sect. 4 presents studies of propagation models for OFDM under severe multipath conditions. Section 5 presents studies on UWB propagation measurement and propagation models.

\section{Classification of Multipath Propagation Models against Frequency Bandwidth}

\section{$2.1 \quad \mathrm{NB}, \mathrm{WB}$ and UWB}

The multipath propagation environment consisting of a group of reflected, scattered, and diffracted waves, as shown in Fig. 1, can be expressed using the impulse response, $h$, as

$$
\begin{aligned}
h\left(t, \tau, f, \theta_{r}, \theta_{t}\right)= & \sum_{i=1} a_{i}(t, f) \delta\left\{\tau-\tau_{i}(t)\right\} \delta\left\{\theta_{r}-\theta_{r, i}(t)\right\} \\
& \delta\left\{\theta_{t}-\theta_{t, i}(t)\right\}
\end{aligned}
$$

where $a_{i}$ is the complex amplitude as a function of time $(t)$ and frequency $(f), \tau_{i}$ is time delay, $\theta_{t, i}$ is angle of departure (AOD), $\theta_{r, i}$ is angle of arrival (AOA) of each path (or elementary wave) $i$, and $\delta$ is the delta function. In the case 


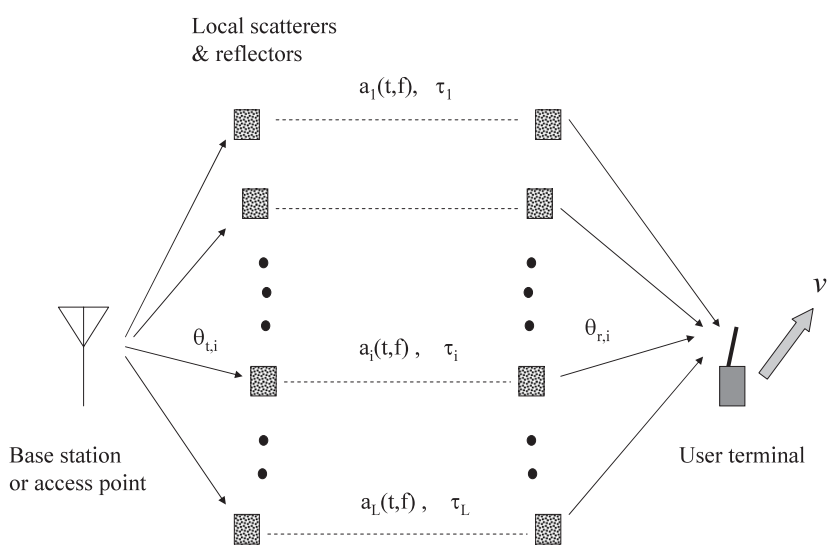

Fig. 1 Multipath propagation environment.

of narrowband (NB) transmission, the delay spread of the propagation channel $\left(\sigma_{\tau}\right)$ is considerably smaller than the symbol period of the modulated signal $\left(T_{\mathrm{s}}\right)$. The transfer function, which is given by Fourier transformation of impulse response, in the signal band with the carrier frequency of $f_{\mathrm{c}}$ exhibits no frequency dependence, so that the fading environment is frequency-flat. To focus the difference on frequency behavior, the impulse response of the channel after integrating over $\theta_{t, i}$ and $\theta_{r, i}$ variables of Eq. (1) becomes

$$
\begin{aligned}
& h_{\mathrm{NB}}(t, \tau, f)\left(\equiv \iint h\left(t, \tau, f, \theta_{r}, \theta_{t}\right) d \theta_{r} d \theta_{t}\right) \\
& =\sum_{i=1} a_{i}\left(t, f_{\mathrm{c}}\right) \delta(\tau) \quad\left(\sigma_{\tau} \ll T_{\mathrm{s}}\right)
\end{aligned}
$$

In wideband (WB) transmission, the delay spread of the propagation channel is nearly equal to or greater than the symbol length of the modulated signal. The transfer function in the band has a frequency-varying characteristic and exhibits frequency-selective fading. However, the transfer function of the elementary wave itself can be considered as having no frequency characteristic. In this case, the impulse response is given as follows:

$$
h_{\mathrm{WB}}(t, \tau, f)=\sum_{i=1} a_{i}\left(t, f_{\mathrm{c}}\right) \delta\left\{\tau-\tau_{i}(t)\right\} \quad\left(\sigma_{\tau} \geq T_{\mathrm{s}}\right)
$$

The Federal Communication Committee (FCC) defines ultra wideband (UWB) signals as "signals with either a large relative bandwidth (typically, larger than $20 \%$ ), or a large absolute bandwidth (> 500 MHz)" [19], [20]. As well as this definition, this paper considers ultla wideband wave propagation, where the frequency characteristic of the elementary wave itself must be considered in transmission, including the recently proposed wireless baseband transmission [21]. In fact, the basic propagation phenomena of reflection, diffraction, and scattering have respective frequency characteristics. In particular, the frequency characteristic of diffraction appears even in a comparatively narrower band than those of reflection and scattering [8], [9], [22]. In this case, the impulse response for UWB channel which is similar to Eq. (1) can be expressed as

$$
h_{\mathrm{UWB}}(t, \tau, f)=\sum_{i=1} a_{i}(t, f) \delta\left\{\tau-\tau_{i}(t)\right\}
$$

Time $t$, delay $\tau$, and frequency $f$ are treated independently in each equation. This expression is the easiest to use in practice. In Refs. [8] and [9], instead of frequency $f$, time-domain expression, which has the similar meaning as Eq. (4), used as follows.

$$
h_{r, \mathrm{UWB}}(t, \tau)=\sum_{i=1} A_{i}(t) \chi_{i}(t, \tau) \otimes \delta\left\{\tau-\tau_{i}(t)\right\}
$$

where $A_{i}$ is path amplitude, $\chi_{i}$ denotes the time-varying distortion of the $i$-th echo due to the frequency selectivity of the interactions with the environment, and means convolution integral. All values in the equation, namely, $h_{r, \mathrm{UwB}}, A_{i}$, and $\chi_{i}$ are real values, and $h_{r, \mathrm{UWB}}$ is corresponding to $h_{r}$ in Eq. (7b) of Sect. 2.2.

Equation (1) expresses the impulse response in a momentary propagation environment with the functions of delay, path angles, and time variations. For statistical expression of multipath environment, a delay power profile (hereafter, delay profile) is used for delay, an angular power profile for AOA and AOD characteristics, and a power spectrum for time-varying characteristics.

\subsection{Equivalent Low-Pass System and Real Number Sys- tem}

According to the classification in the previous section, modulated signal for NB and WB is a band-pass signal with a carrier frequency of $f_{\mathrm{c}}$. This signal can be converted to a complex signal with the center frequency of $f=0$ which is called the equivalent low-pass system (or baseband system) [23]. If the transmit signal for a WB system is $s(t)$ and the thermal noise at the receiver is $n(t)$, the received signal $r(t)$ can be expressed as

$$
r(t)=h(t) \otimes s(t)+n(t)
$$

All quantities including the impulse response $h(t)$ are given by complex numbers in the equivalent low-pass system.

In impulse radio (IR) [24], [25] of UWB or wireless baseband transmission (WBT) [21], [26] for the direct transmission of baseband continuous forms from an antenna, real-number signals are transmitted because there are no carrier waves. In this case, a transmission channel must be expressed by a real number. The received signal $r_{r}(t)$, transmitting signal $s_{r}(t)$, thermal noise $n_{r}(t)$, and impulse response $h_{r}(t)$ are also real numbers and can be expressed as

$$
\begin{aligned}
& r_{r}(t)=h_{r}(t) \otimes s_{r}(t)+n_{r}(t) \\
& h_{r}(t)=2 \operatorname{Re}\left[\mathcal{F}^{-1}\left\{H^{+}(f)\right\}\right]
\end{aligned}
$$

where $\operatorname{Re}[x]$ shows the real part of complex number $x$, and $\mathcal{F}^{-1}$ means the inverse Fourier transformation.

Usually, UWB propagation channel characteristics are measured in the frequency domain such as using a frequency-sweep type vector network analyzer (VNA). In that case, the measured channel characteristics are often 


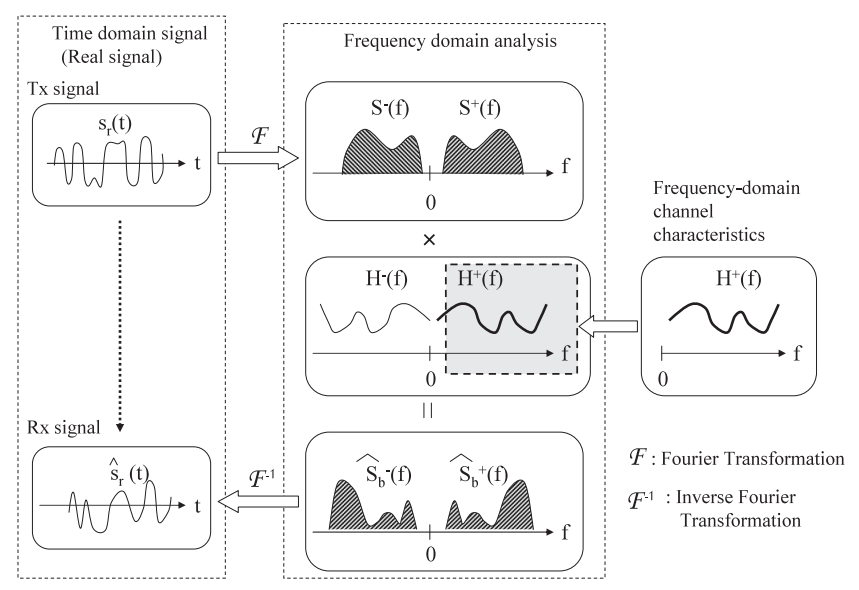

(a) Frequency-domain analysis of time-domain real signal

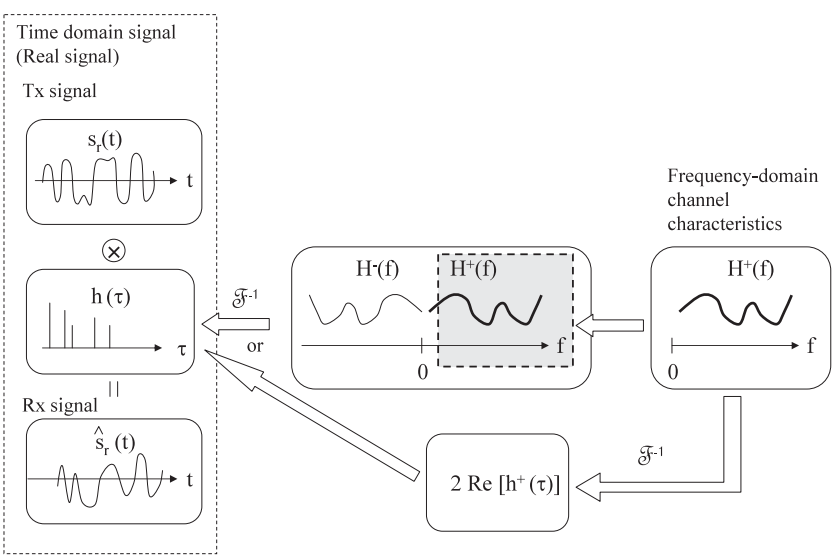

(b) Time-domain analysis of real signal

Fig. 2 Analysis of UWB signal such as impulse radio or wireless baseband transmission by using channel characteristics based on frequencydomain measurement.

given by complex-number signal as a function of frequency denoted by $H^{+}(f)$. If complex channel characteristics are applied to a real-number Tx/Rx signal, $h_{r}$ is converted into a real number (Eq. (7)) or the real-number Tx signal is once converted into a frequency domain signal and analyzed it in the frequency region. Figure 2 shows two kinds of analysis methods of UWB signal using channel characteristics based on frequency-domain measurement.

\section{Spatio-Temporal Propagation Channel Model}

A spatio-temporal propagation model is required for the receiving or transmitting of WB signals using an array antenna. There have been several books of this topic [1]-[3] and it cannot be fully treated in this limited survey. Here, some recent topics are presented.

\subsection{Statistical Model for Angular Power Profile}

Widely accepted mobile propagation model on angular power profile of incident waves adopts an environment where multipath waves arrive at a mobile terminal from a wide range of angles and at a base station from a limited

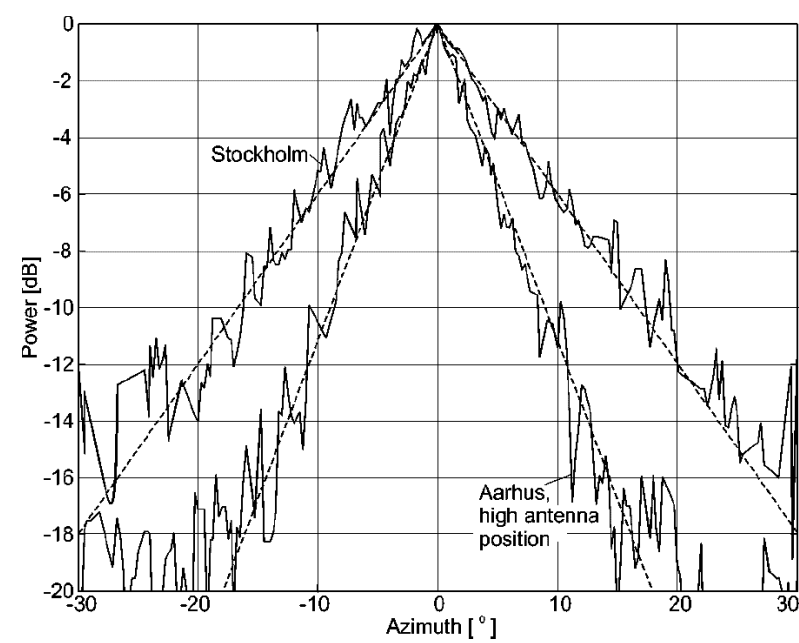

Fig. 3 Estimated angular power profiles from measurements [32]. (Pedersen et al., (c)2000 IEEE)

range of angles, mainly in the direction of the mobile terminal [27], [28]. Since the angular power profile and spatial correlation function can be connected by the Fourierlike transformation so that angular power profiles were estimated from spatial correlation measurements until 1990s. Resultantly, the angular power profile at around a base station (BS) has been modeled as a normal distribution [29] while that around a mobile station (MS) as a uniform distribution because it is difficult to identify the profile precisely from the space correlation measurement. The relationship between the angular power profile $\Omega(\theta)$ and space correlation characteristics of power variations $\rho_{P}(d)$ for BS is

$$
\begin{aligned}
& \Omega(\theta) \propto \frac{1}{\sqrt{2 \pi} \sigma_{\theta}} \exp \left\{-\frac{\left(\theta-\theta_{0}\right)^{2}}{2 \sigma_{\theta}^{2}}\right\} \\
& \rho_{P}(d) \approx \exp \left[-\left(k \sigma_{\theta} d \sin \theta_{0}\right)^{2}\right]
\end{aligned}
$$

where $\theta$ is angle of direction measured from the baseline direction, $\theta_{0}$ is the angle of the mobile terminal direction, $\sigma_{\theta}$ is the standard variation of angular power spread, $d$ is the distance of two points, and $k$ is the wave number of the carrier frequency signal (note that $k$ is other definition in other sections).

Recently, using an array antenna, a super-highresolution AOA estimation method based on maximum likelihood estimation method such as SAGE has been developed and the angular power profile can now be identified in detail. Consequently, the Laplacian distribution expressed by the following equation has been found to approximate the actual angular profile well at the base station side [30]-[34]:

$$
\Omega(\theta) \propto \frac{1}{\sqrt{2} \sigma_{\theta}} \exp \left\{-\frac{\sqrt{2}\left|\theta-\theta_{0}\right|}{\sigma_{\theta}}\right\}
$$

Figure 3 shows measured angular power profile for base station in city environments [32]. The angular distribution data in both "Aathus (Denmark)" and "Stockholm (Sweden)" were acquired in each non-line-of-sight (NLOS) 


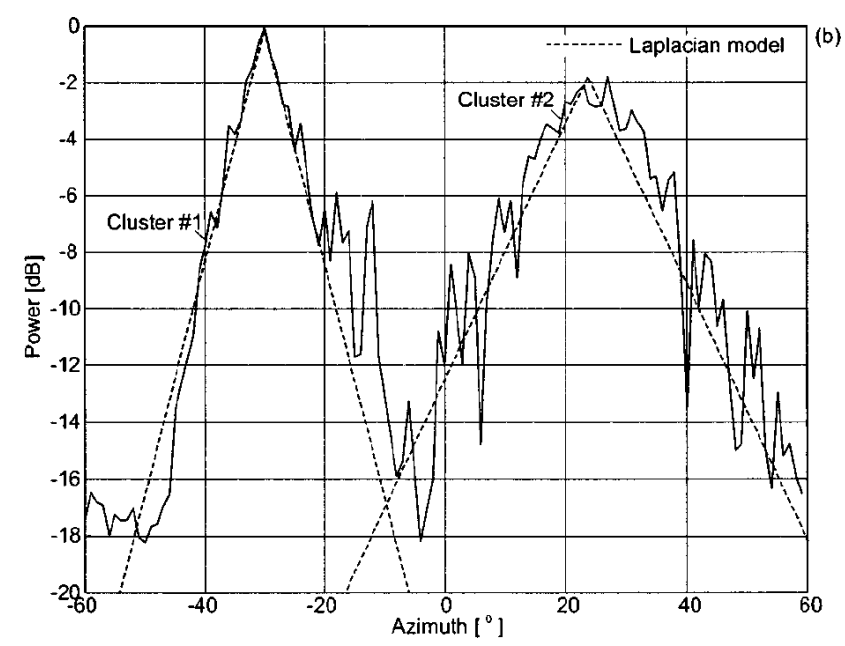

Fig. 4 Example of angular power profile composed of two clusters [32]. (Pedersen et al., (c)2000 IEEE)

environment. The environment of Aathus has four- to sixstoried buildings and roads that run irregularly. That of Stockholm also has four- to six-storied buildings, but has a slightly rolling terrain. In Aathus, the base station antenna height is $32 \mathrm{~m}$ above the ground, about $10 \mathrm{~m}$ higher than the average building height. In Stockholm, however, the base station antenna height is $21 \mathrm{~m}$, almost equal to the average building height. Signal of $1.8-\mathrm{GHz}$ band with $4.096 \mathrm{Mcps}$ which corresponds to the bandwidth of W-CDAM signal was used for measurement and a plane array antenna of $10 \times 4$ elements was used for angular measurements. The arrival angle was estimated by the SAGE algorithm, which is a high-resolution estimation algorithm based on the principle of maximum likelihood estimation. From the figure, we see that the measured values match the Laplacian distribution well. With angular spread data in three environments, including another data at the BS antenna height of $20 \mathrm{~m}$ in Aathus (Aathus low antenna position), the medians (50\% values) of the cumulative distribution were compared and the angular spread $\left(\sigma_{\theta}\right)$ was reported to be about $5^{\circ}$ for a high base station and about $10^{\circ}$ to $12^{\circ}$ for an antenna at about the average building height. When $90 \%$ values were compared, the spread was reported to be about $14^{\circ}$ for a high base station and about $22^{\circ}$ to $26^{\circ}$ for an antenna at the average building height.

If the urban structures are statistically uniform, they can be expressed by a single Laplacian distribution as above. As a special case, a distribution consisting of multiple clusters can be acquired as shown in Fig. 4 [32]. In this example, the area is one of non-uniform urban environments consisting of a mixture of open areas and dense buildup zones with a large variety of different building heights.

Reference [34] reports measurements in a city environment to examine the relationship between street direction and scattered area. This experiment was conducted using a 16-element horizontal linear array antenna on the base station side with $60 \mathrm{~m}$ in height and the mobile station with

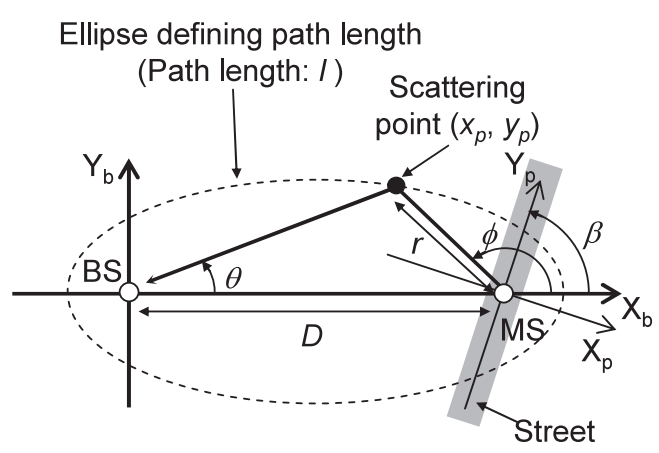

Relative path length: $\Delta I=I-D$

(a) Coordinate transformation along a street.

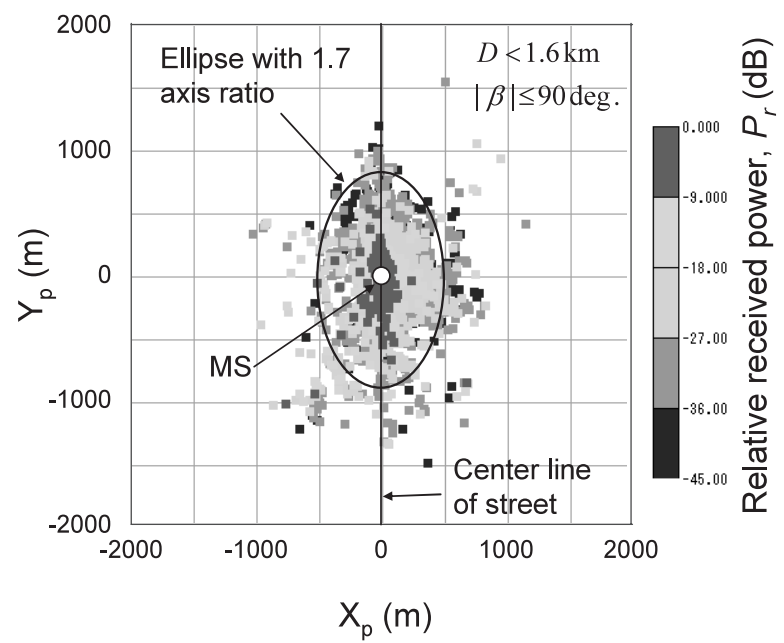

(b) Two-dimensional scattering power distribution in the $X_{p}-Y_{p}$ coordinate system.

Fig. 5 Two-dimensional scattering power distribution in the $X_{p}-Y_{p}$ coordinate system in which the $Y_{p}$ axis is parallel to the street [34]. (Imai and Taga, (c)2006 IEEE)

$3.5 \mathrm{~m}$ in height. In this experiment, the angular power profile also exhibited a Laplacian pattern and the angular spread was $7.3^{\circ}$. By data analysis, this experiment clarified that the scattered area around a mobile terminal can be expressed as an ellipse with its major axis along the street, as shown in Fig. 5 .

When the angular distribution is a Laplacian distribution, the space correlation can be expressed as

$$
\rho_{P}(d) \approx \frac{1}{\left(1+\frac{k^{2} d^{2} \sigma_{\theta}^{2} \sin ^{2} \theta_{0}^{2}}{2}\right)^{2}}
$$

Figure 6 compares a calculation result of the space correlation compared with that for a normal distribution with the same angular spread. Until now, the space diversity effect and other related spatial signal processing have mostly been calculated assuming a normal distribution for the angular power profile. If the Laplacian distribution represents the actual environment, however, the theoretical model of space diversity may require slight modification. Since the differences are not large for higher correlation part in Fig. 6, it may be considered that the most conventional study results 


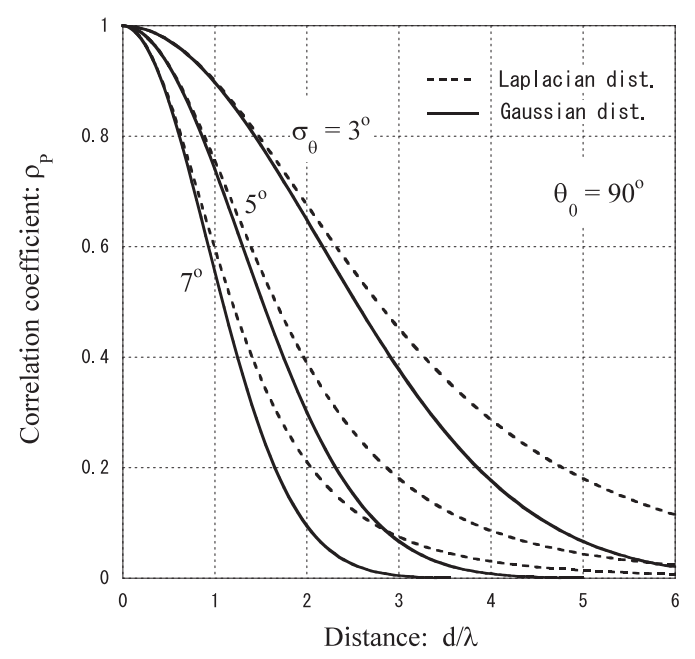

Fig. 6 Spatial correlation characteristics calculated based on both Gaussian profile and Laplacian profile.

will not be necessary to change significantly.

The above is an NB model based on the assumption that the frequency characteristic should not change in the band. The angular profile may not be very dependent on frequency but its influence on WB signals needs to be considered because space correlation is determined as a function of the wavelength (i.e. frequency). This influence is not significant for WB signals [35], but should be considered carefully for UWB signals.

\subsection{Delay Profile}

For WB signal transmission, the delay profiles of propagation channels necessary for the evaluation of its transmission characteristics have been measured and an enormous amount of data has been accumulated. From the viewpoint of modeling, however, delay profiles both in indoor and outdoor environments can be described by the exponentialdecay-type function expressed as

$$
p(\tau) \propto \frac{1}{\sigma_{\tau}} \exp \left(-\frac{\tau}{\sigma_{\tau}}\right)
$$

In an indoor environment, particularly in a multipathrich environment surrounded by very reflective walls, the delay profile is dense with many elementary waves [36], [37]. In outdoor environments, on the other hand, the delay profile is often sparse with fewer elementary waves [32], [38]. As a measure of delay dispersion in a statistical sense, the term "delay spread" which is defined by the standard deviation of power delay profile is used. The delay spread varies greatly depending on propagation environment, and from $10 \mathrm{~ns}$ to $100 \mathrm{~ns}$ in indoors and from $100 \mathrm{~ns}$ to several micro-seconds in outdoors in most of cases.

In a multi-cluster case, as shown in Fig. 4, a delay profile is composed of several sub-delay profiles with different averaged delays [32]. For modeling, each sub-delay profile itself employs an exponential-function delay profile in general [39], [40].

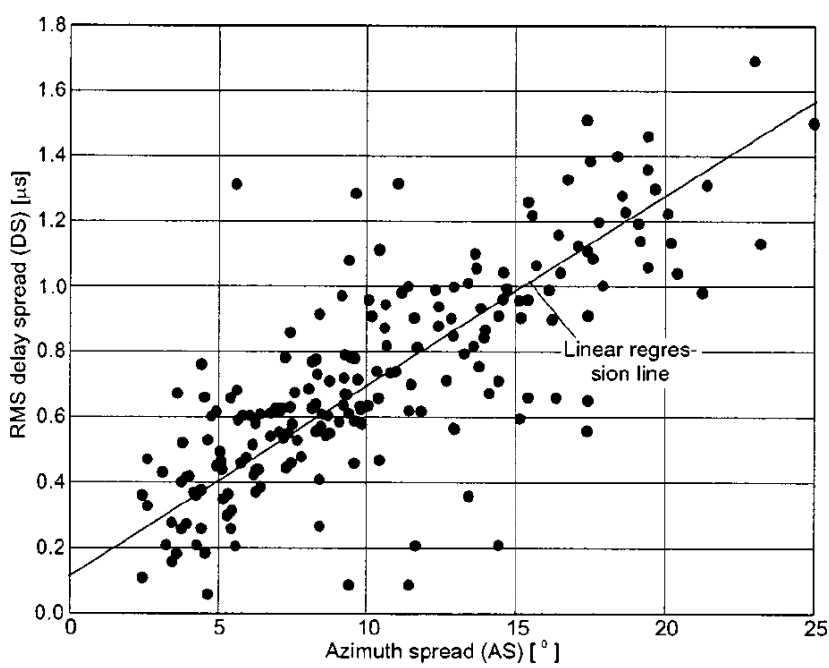

Fig. 7 Scatter plot of the estimated angular spread and delay spread [32]. (Pedersen et al., (c)2000 IEEE)

In the RAKE combining scheme for CDMA that captures separated delayed-waves by means of path diversity, statistical analysis and modeling by which the separated delayed waves are rearranged in descending order of intensity have been investigated [41].

\subsection{Correlation between Angular Spread and Delay Spread}

The individual characteristics of arrival angle distribution and delay profile are described in 3.1 and 3.2. For WB signal reception (or transmission) by an array antenna, the relationship between angular power distribution $\Omega(\theta)$ and delay profile $p(\tau)$ is important. In most measurements so far, the following independency for two-dimensional profile on $\theta$ and $\tau$ was identified:

$$
P(\theta, \tau) \propto \Omega(\theta) p(\tau)
$$

In the aforementioned Ref.[32], using the simultaneous measurement data of angular profile and delay profile, it was identified that two profiles are not mutually affected for angular profiles at various delay bins $(\tau \sim \tau+\Delta \tau)$ and delay profiles at various arrival angle bins $(\theta \sim \theta+\Delta \theta)$. Thus, the angular distribution and delay profile can be handled independently in the scattering characteristic of a single cluster as given by Eq. (13). This, however, does not mean that there is no correlation between angular spread and delay spread. The measurement results in Ref. [32] indicate a clear correlation (correlation factor 0.72 ) between the parameters as shown in Fig. 7.

\subsection{Relation between Space Correlation Characteristics and Space Diversity Effect}

Space correlation characteristics can be transformed from the angular power profile by Fourier-like transformation. For space diversity in reception using an array antenna, the 


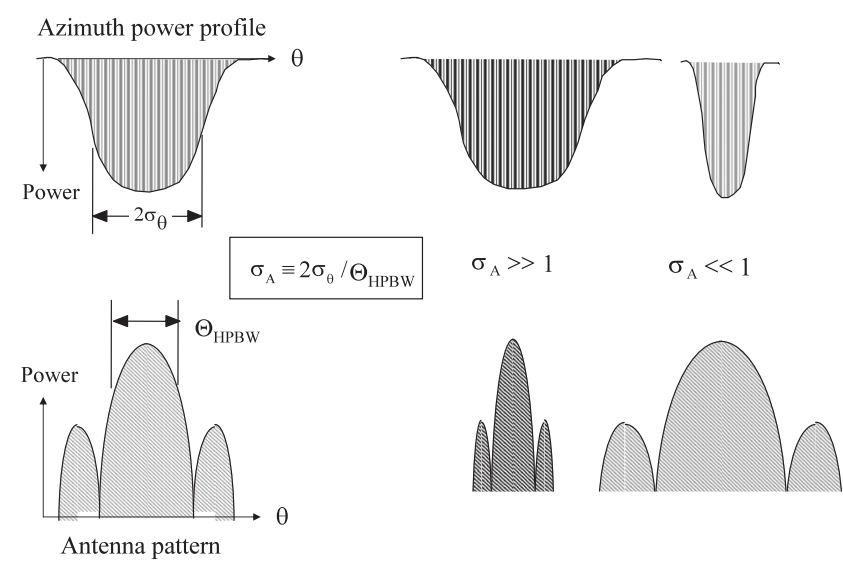

Fig. 8 Angular spread vs. antenna beamwidth for space diversity performance analysis.

array spacing must be greater than the coherent length which gives $\rho_{P}$ of 0.5 for example to prevent correlation from gaining sufficient diversity effect. The method of investigation in Ref. [42] is introduced here. As Fig. 8 shows, the object is to clarify how the relationship between the angular spread ( $2 \sigma_{\theta}$ in this case) and the half-power beamwidth $\Theta_{\text {HPBW }}$ of the antenna array pattern influences the space diversity effect.

An array with $M$ elements arranged linearly with a spacing $d$ is considered. The angular power profile is the normal distribution, and the number of elementary waves in this angle range is sufficiently large, namely, in a dense multipath environment. The space correlation characteristics $\rho_{m n}$ between complex amplitudes for elements $m$ and $n$ can be expressed as

$$
\rho_{m n}=\exp \left[j k(n-m) d \cos \theta_{0}-\frac{k^{2}\{(n-m) d\}^{2} \sin ^{2} \theta_{0} \sigma_{\theta}^{2}}{2}\right]
$$

The correlation coefficient in the equation has a complex value, and that for power variation in Eq. (9) can be obtained by $\left|\rho_{m n}\right|^{2}$ with replacing $(n-m) d$ by $d$. A relative angular spread parameter $\sigma_{\mathrm{A}}$ defined by the following equation is introduced.

$$
\sigma_{\mathrm{A}} \equiv 2 \sigma_{\theta} / \Theta_{\mathrm{HPBW}}
$$

With the eigenvalue $\lambda_{s m}(m=1,2, \ldots, M)$ of the correlation matrix, the components of which is given by Eq. (14), the relationship between the eigenvalue normalized by $M \Gamma_{0}$ $\left(\Gamma_{0}\right.$ : the averaged carrier-to-noise power ratio $\left.(\mathrm{CNR})\right)$ and the parameter $\sigma_{\mathrm{A}}$ is given for the cases of $M=2,4,8$ in Fig. 9. When $\sigma_{\mathrm{A}}$ is smaller than 0.1 , there is only one significant eigenvalue in all cases and spatial signal processing effect does not appear. When $\sigma_{\mathrm{A}}$ is about 1 , where the angular spread $\left(2 \sigma_{\theta}\right)$ and the beamwidth of the antenna are equal, the largest eigenvalue and the second largest eigenvalue are almost equal. As $\sigma_{\mathrm{A}}$ becomes greater than 1 or the antenna spacing increases, all eigenvalues approach the same value and the space signal processing capacity by the number of

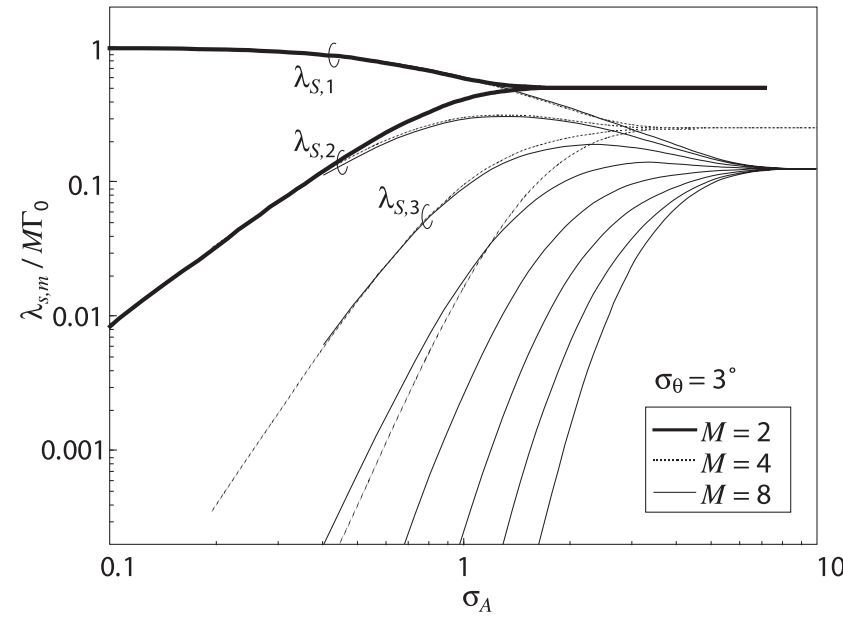

Fig. 9 Normalized eigenvalue characteristics as a function of the parameter $\sigma_{\mathrm{A}}[42]$.

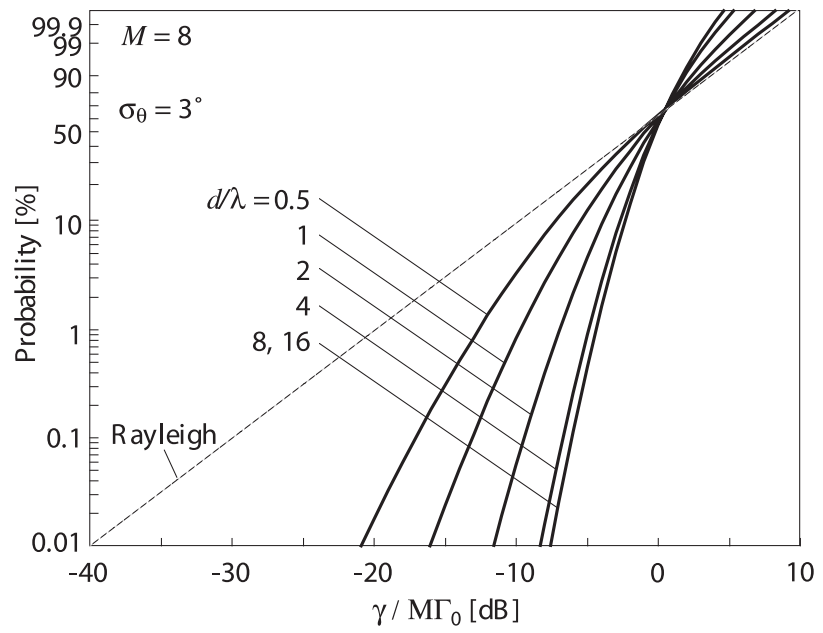

Fig. 10 Diversity effects vs. antenna spacing for $M=8$ and $\sigma_{\theta}=3 \mathrm{deg}$. [42].

antenna elements or the effect of diversity become maximal. The CNR after the maximal ratio combining by space diversity can be calculated by using the following equation [43]:

$$
f(\gamma)=\frac{1}{\prod_{m=1}^{M} \Gamma_{m}} \sum_{m=1}^{M} \frac{\exp \left(-\gamma / \Gamma_{m}\right)}{\prod_{\substack{n \neq m \\ n=1}}^{M}\left(\frac{1}{\Gamma_{n}}-\frac{1}{\Gamma_{m}}\right)} \quad \Gamma_{m} \equiv \lambda_{s m} \Gamma_{0}
$$

where $\Gamma_{0}$ is CNR for $M=1$.

Using the antenna spacing as a parameter, Fig. 10 shows the effect of space diversity under the conditions of $\sigma_{\theta}=3^{\circ}$ and $M=8$ [42]. When the antenna spacing normalized by wavelength is $0.5,1,2,4$, and 8 , the value of $\sigma_{\mathrm{A}}$ is $0.40,0.81,1.6,3.2$, and 6.4 , respectively, from Eq. (15) in this case. As a matter of course, the effect becomes greater as the antenna spacing increases. When the antenna spacing is equal to about four wave length (i.e. $\sigma_{\mathrm{A}}=3.2$ ) or more, the effect becomes almost saturated. This can also be predicted from the eigenvalue distribution shown in Fig. 9 where eight 
eigenvalues for $M=8$ become closer.

\subsection{Power Variation Statistics of WB Signals}

It is well known that the power variation of WB signals in fading decreases with increasing the bandwidth. Also in RAKE combining for CDMA, increasing the number of fingers produces signals with less fluctuation. The level fluctuations of WB signals has been analyzed by simulation or simulation-based modeling with some theoretical discussion [44], [45], but none of the methods had theoretically completed. Regarding this problem, a theoretical model based on eigenvalue analysis in frequency domain and linked to the theory of frequency diversity was presented [46], [47]. This theoretical model [47] is introduced here.

The object of the model is to determine the probability distribution of the received power variations. The power variation is that of signals whose bandwidth is limited by the ideal filter $-B / 2 \leq f \leq B / 2$. The received power $P_{r}(t)$ is

$$
P_{r}(t)=\int_{-B / 2}^{B / 2} p_{r}(f, t) d f
$$

where $p_{r}(f, t)$ is the received power density at frequency $f$ and time $t$. The value above is the average over a time period sufficiently longer than the symbol length $T_{\mathrm{s}}$ of the modulated signal and considerably shorter than the averaged period of fading fluctuation due to Doppler spread. If the averaged power spectrum of transmitting signals is uniform in the band, the power spectral density of a receiving signal after the ideal filter can be represented by using the transfer function $|T(f)|^{2}$.

To link this analysis to the theory of the frequency diversity composed of branches having correlations, the entire band is divided into $K$ sub-bands. The value of $K$ is determined so that the frequency characteristic will be flat in each sub-band. The total number of subbands $K$ should be sufficiently larger than $B \sigma_{\tau}$. If the momentary average power of a signal in sub-band $k$ is $P_{k}(t)$ and there are sufficient narrow bands, $P_{k}$ takes the same exponential distribution (i.e. amplitude of Rayleigh distribution). The received power $P_{r}(t)$ can be expressed as

$$
\begin{aligned}
& P_{r}(t)=\sum_{k=1}^{K} P_{k}(t) \\
& P_{k}(t)=p_{r}\left\{f_{\min }+(k-1) \Delta f, t\right\} \Delta f
\end{aligned}
$$

where $\Delta f$ is the bandwidth of a sub-band $(=B / K)$ and $f_{\min }$ is the minimum frequency $(=-B / 2)$ of the band. If only the defined condition is satisfied, the received power $P_{r}(t)$ does not depend on $K$. Then the correlation matrix $\boldsymbol{R}_{f f}$ between sub-bands $m$ and $n$ can be expressed as

$$
\begin{aligned}
& \boldsymbol{R}_{f f}=\left[R_{m n}\right] \\
& R_{m n}=\frac{1}{K} \int_{0}^{\infty} p(\tau) \exp \{-j 2 \pi \Delta f(n-m) \tau\} d \tau
\end{aligned}
$$

where $p(\tau)$ is the delay profile considered here. In an exponential delay profile, $R_{m n}$ can be expressed as

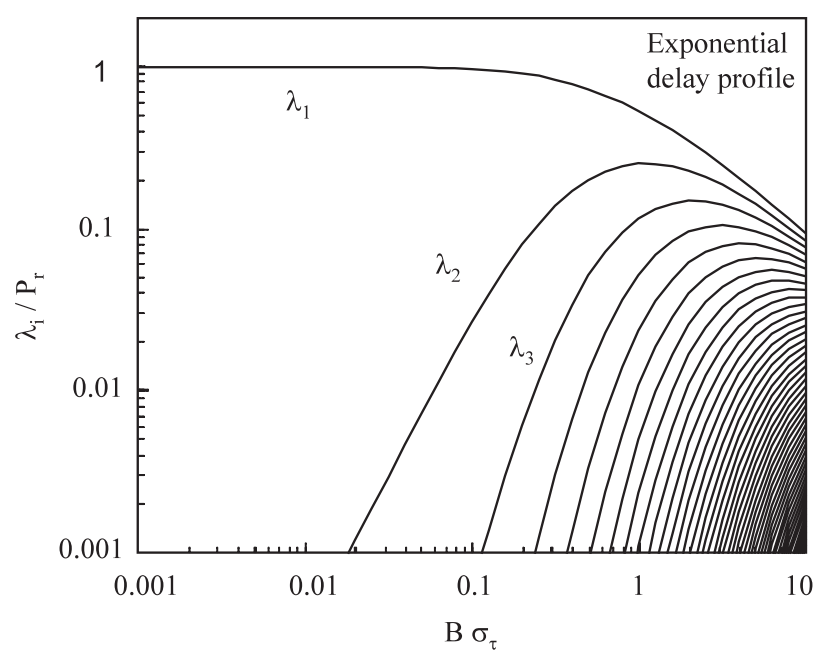

(a) Eigenvalues as a function of $B \sigma_{\tau}$

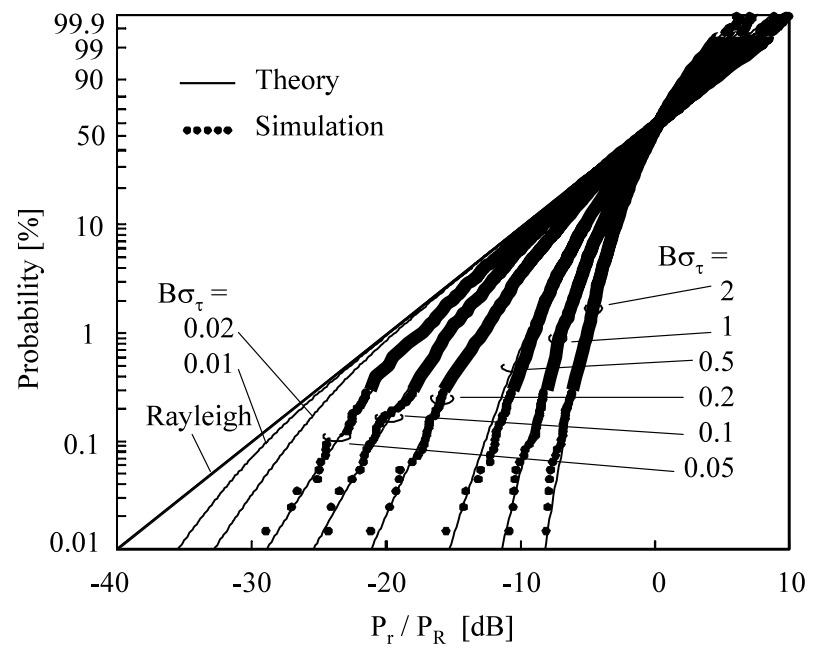

(b) $\mathrm{CDF}$ of power variations for the parameter of $B \sigma_{\tau}$

Fig. 11 CDFs of power variations for $\sigma_{\tau} / T_{\mathrm{s}}$ in exponential delay profile [47].

$$
R_{m n}=\frac{P_{\mathrm{R}}}{K} \frac{1-j 2 \pi(n-m) \sigma_{\tau} \Delta f}{1+\left\{2 \pi(n-m) \sigma_{\tau} \Delta f\right\}^{2}}
$$

where $P_{\mathrm{R}}$ is the average received power $\left(=\left\langle P_{\mathrm{r}}\right\rangle\right)$ over a time much longer than the fading period.

The probability distribution of $P_{\mathrm{r}}$ here can be resolved to the probability distribution of the SNR acquired by the maximal ratio combining of signals having correlations between branches in diversity combining introduced in the previous section. More specifically, the eigenvalues of the correlation matrix $\boldsymbol{R}_{f f}$, the elements of which are given by Eq. (20), is calculated first. This consequently determines the positive real-number eigenvalues $\lambda_{i}(i=1,2, \ldots, K)$. From the $K$ values arranged in descending order, an appropriate number of top $K_{0}$ values (typically, the number of eigenvalues greater than $1 / 100$ of the largest eigenvalue) is selected, and variables $\gamma, \Gamma_{m}$ and $M$ in Eq. (16) are replaced by $P_{r}, \lambda_{i}$ (with $m$ by $i$ ), and $K_{0}$, respectively. Finally, by calculating Eq. (16) with replaced variables as mentioned above, the probability density function of power variations 
of WB signals under arbitrary delay profile environment can be obtained theoretically.

Figure 11 shows the cumulative distribution of the power variation with $B \sigma_{\tau}$ as a parameter in an environment of an exponential-function delay profile with corresponding eigenvalue characteristics [47]. As mentioned at the beginning of this section, fluctuations are suppressed more as the bandwidth expands. The calculations are based on the assumption that the number of paths is sufficiently large, namely, dense delay profile. If the number of paths is not sufficient, the form of the cumulative distribution differs depending on the delay profile because the number of significant eigenvalues decreases. This means that the number of eigenvalues will never exceed the number of paths even if the number of sub-bands, $K$, becomes larger. For details, see Ref. [47].

\section{Propagation Modeling for OFDM}

\subsection{Has OFDM Overcome the Propagation Problem?}

The difference between cable communication and radio communication is that radio communication, particularly mobile communication, has a problem with signal deterioration due to propagation effects. Therefore, it is reasonable to say that the history of radio communication technology development is the history of the countermeasure for wave propagation impairments. These days, the requirements of WB transmission are growing and studies are focusing on the problem of multipath propagation delay affecting the transmission. Meanwhile, as OFDM resistant to multipath delay spread becomes popular in communication systems, it is believed that the propagation problem has been solved. Is this correct?

Since WB signal is transmitted through a number of narrowband channels in OFDM, the scheme is substantially resistant to delay spread (or inter-symbol interference: ISI). For completing the resistance to ISI, OFDM has a mechanism called guard interval with cyclic prefix (time length $\left.T_{\mathrm{GI}}\right)$. Since the "delay spread: $\sigma_{\tau}$," represents the standard deviation of the delay dispersion, the tail of the delay profile for consideration is several times greater. Therefore, $\sigma_{\tau} \ll T_{\mathrm{GI}}$ (condition 1) is a necessary condition for suppressing ISI. Block-processed signals are sent through the time of the effective symbol period $T_{\mathrm{s}}$. From the viewpoint of the transmission efficiency, however, $T_{\mathrm{GI}} \ll T_{\mathrm{s}}$ (condition 2) is preferable, and is another necessary condition. An OFDM signal block-processed in units of $T_{\mathrm{S}}$ loses its orthogonality and results in errors called inter-carrier interference (ICI) if the environment changes during the time. If the minimum cycle of time fluctuation by Doppler spread is $T_{\mathrm{f}}$ $\left(=1 / f_{\mathrm{D}}\right)\left(f_{\mathrm{D}}\right.$ : maximum Doppler frequency $)$, the condition $T_{\mathrm{s}} \ll T_{\mathrm{f}}$ (condition 3 ) prevents this from occurring errors due to ICI. In general, $T_{\mathrm{s}}$ should be $1 / 100$ of $T_{\mathrm{f}}$ or smaller. These three necessary conditions can be summarized by the relationship shown in Fig. 12 and expressed as follows:

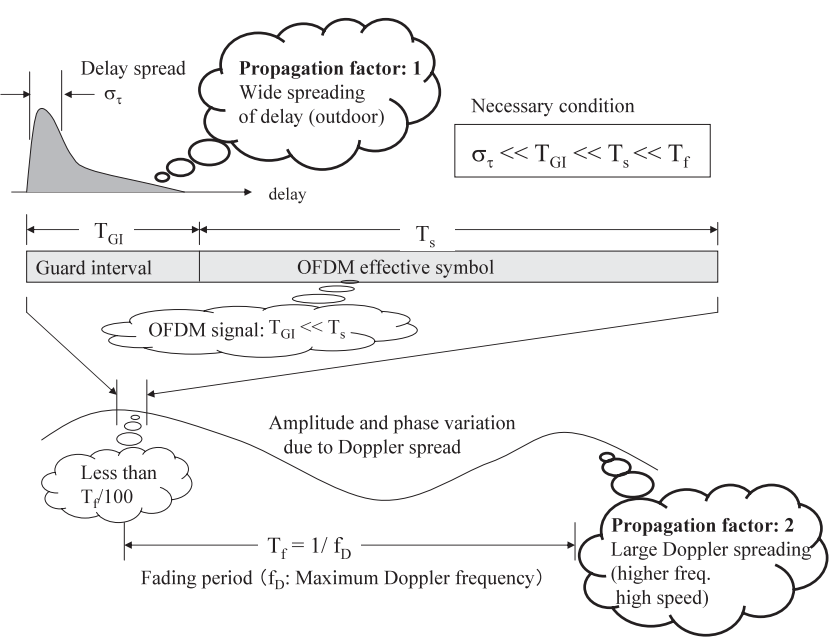

Fig. 12 Two propagation factors affecting OFDM transmission characteristics.

$$
\underbrace{\sigma_{\tau} \ll T_{\mathrm{GI}} \ll T_{\mathrm{S}}}_{\text {ISI }} \underbrace{\ll T_{\mathrm{f}}}_{\text {ICI }}
$$

OFDM is used for wireless LAN (IEEE 802.11a) and terrestrial digital broadcasting systems (ISDB-T). Since $T_{\mathrm{GI}}$ is $800 \mathrm{~ns}$ and $T_{\mathrm{s}}$ is $3.2 \mu \mathrm{s}$ for the wireless LAN, assuming an indoor environment $\left(\sigma_{\tau}\right.$ : order of 10-100 ns), the above conditions are mostly satisfied. For terrestrial digital broadcasting system, setting a long guard interval $\left(T_{\mathrm{GI}}=126 \mu \mathrm{s}\right)$ makes $T_{\mathrm{s}}$ as long as $1.008 \mathrm{~ms}$. Thus, condition 3 seems necessary to reconsider, however, the conditions are totally satisfied. Based on this, OFDM fully meets the requirements for these two services.

For future mobile communications, on the other hand, a delay spread of about $2 \mu$ s must be estimated because the service will be provided in outdoor environments. The guard interval should then be about $10 \mu$ s or longer. If an operation of $5-\mathrm{GHz}$ frequency band is assumed, the service may also be applied to high-speed mobile terminals of $30 \mathrm{~m} / \mathrm{s}(=108 \mathrm{~km} / \mathrm{h})$, for example. Since $f_{\mathrm{D}}$ is up to $500 \mathrm{~Hz}$ ( $T_{\mathrm{f}}$ of $2 \mathrm{~ms}$ ) for the service, $T_{\mathrm{s}}$ should be suppressed to about $20 \mu \mathrm{s}$ or less. Then the realization of efficient transmission in the condition 2 is affected seriously. Now we understand that the robustness of OFDM to multipath propagation is only a myth. Difficulty for determining appropriate OFDM parameter values will emerge soon from the pincers movement of two different propagation phenomena.

The next two sections deal with the relationship between propagation factors and the OFDM transmission characteristic from the above propagation viewpoint.

4.2 Wave Propagation Model for OFDM Transmission Characteristic Evaluation where the Delay Profile Exceeds the Guard Interval

OFDM is a very powerful transmission scheme effective for WB systems under frequency-selective fading environments. It is a mechanism for transmitting WB signals by 
dividing them into many NB signals, as mentioned above. Therefore, WB signals can be handled as NB signals in each subchannel under the following conditions: 1) the band is divided into sufficiently narrow bands compared with the coherent bandwidth and 2) the guard interval is set greater than the maximum delay of the delay profile. Under these conditions, even if the fading over total bandwidth is frequencyselective, we can handle each signal of each subband NB signal. Theory of NB signal transmission is well developed so far, this is not our concern in this paper. This section introduces a propagation model for evaluating the transmission characteristic in a very severe propagation environment where the maximum delay exceeds the OFDM guard interval. Although the degradation due to this effect can be understood qualitatively, quantitative analysis might be difficult because the effect is not only ISI but also ICI due to the deterioration of orthoganality. The evaluation of the transmission characteristic in an environment of large delay beyond the guard interval has been roughly verified by computer simulation or too much simplified calculations [48]. Under this situation, a theoretical model which combines wave propagation parameters and system parameters universally has been developed in Ref. [49]. To the model, we call it "the equivalent transmission-path (ETP) model for OFDM," shortly, "ETP-OFDM model." Hereafter, we introduce this model.

\subsubsection{Equivalent Transformation of Delay Profile for OFDM}

For the statistical evaluation of error occurrence, the bit error rate (BER) is used in general. In an environment of deteriorating wave propagation, the average BER can be calculated by the following basic equation:

$$
P_{e}=\iiint_{x, y, z} f_{p}(x, y, z, \cdots) \cdot P_{0}(x, y, z, \cdots) d x d y d z \cdots
$$

where $P_{0}$ is the BER determined by the functions of the variables $(x, y, z, \ldots)$ prescribing the propagation status and $f_{p}$ is the probability density function (PDF) indicating the probability of the propagation status $(x, y, z, \ldots)$. If only $f_{p}$ is acquired from the propagation phenomenon and $P_{0}$ from each modulation/demodulation scheme, the average BER can be calculated. Although complicated relation between "Propagation" and "Modulation/Demodulation scheme" makes it difficult to understand the digital transmission characteristics intuitively, we see from Eq. (22) that the two are explicitly separated and the physical meaning is very clear.

The equivalent transmission-path model for OFDM (ETP-OFDM) in Ref. [49] applies this concept to the estimation of irreducible errors that occur in an environment where there are delays beyond the guard interval. The ETPOFDM model is based on the ETP model previously developed [50]-[53] that estimates a BER floor value caused by inter-symbol interference (ISI) in single-carrier transmission.

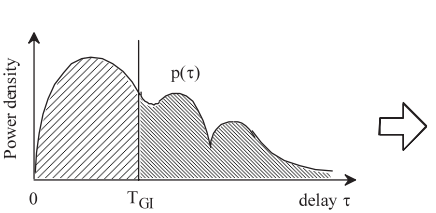

(a)

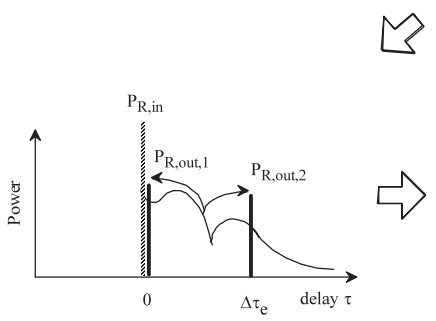

(c)

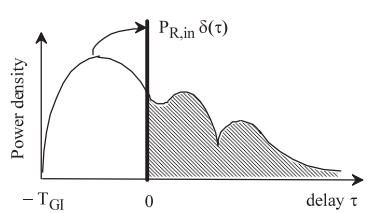

(b)

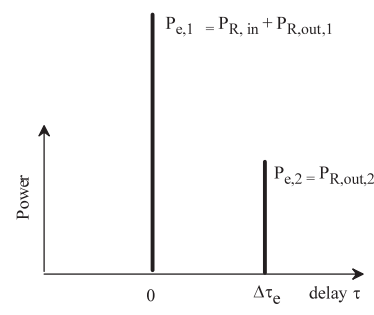

(d)
Fig. 13 Equivalent transformation of power delay profile (development of ETP-OFDM) [49]. (a) A delay profile which exceeds the guard interva (GI)1, (b) Equivalent transformation of the part of delay profile in which the daly is less than GI, (c) The same as (b) but the delay is larger than GI, (d) ETP-OFDM model.

Figure 13 shows the process of obtaining an equivalent two-path model in which we call the ETP-OFDM model in the case of Rayleigh fading [49]. The validity of this equivalent transformation is based on the analysis in Ref. [54] where the translation of instantaneous environment expressed by the impulse response is examined by the similar way. Figure 13(a) shows a typical delay profile, $p(\tau)$, that exceeds the guard interval. In Fig. 13(b), delayed waves within the guard interval are integrated into one wave of delay 0 having average power $P_{\mathrm{R}, \mathrm{in}}$. The delay profile for OFDM analysis, $p_{\text {OFDM }}$, is given by

$$
\begin{aligned}
& p_{\mathrm{OFDM}}\left(\tau ; T_{\mathrm{GI}}\right)=P_{\mathrm{R}, \text { in }} \delta(\tau)+p\left(\tau+T_{\mathrm{GI}}\right) u(\tau) \\
& u(\tau) \equiv\left[\begin{array}{ll}
1 & (\tau>0) \\
0 & (\tau \leq 0)
\end{array}\right. \\
& P_{\mathrm{R}, \text { in }}=\int_{0}^{T_{\mathrm{GI}}} p(\tau) d \tau
\end{aligned}
$$

In Fig. 13(c), delayed waves beyond the guard interval are separated into the delay 0 wave having average power $P_{\text {Rout } 1}$ and the delay $\Delta \tau_{e}$ wave having average power $P_{\text {Rout } 2}$. The power distribution of the delayed wave power $P_{\text {Rout }}$ to $P_{\mathrm{R}, \text { out }, 1}$ and $P_{\mathrm{R}, \text { out }, 2}$ and $\Delta \tau_{e}$ can be determined by the established method of the equivalent transmission-path (ETP) model for single-carrier transmission [50], such that the mean delay and delay spread of the two-path model maintain the same values of those for the delay profile $p(\tau)$ for $\tau>T_{\mathrm{GI}}$. Finally, the equivalent delay profile for the OFDM signal can be expressed as

$$
p_{e}(\tau)=P_{e, 1} \delta(\tau)+P_{e, 2} \delta\left(\tau-\Delta \tau_{e}\right)
$$

Equations for expressing $P_{e, 1}, P_{e, 2}$ and $\Delta \tau_{e}$ in details are given in Ref. [49].

The equivalent impulse response for instantaneous multipath environment can be expressed on subchannel-bysubchannel basis by the form of 


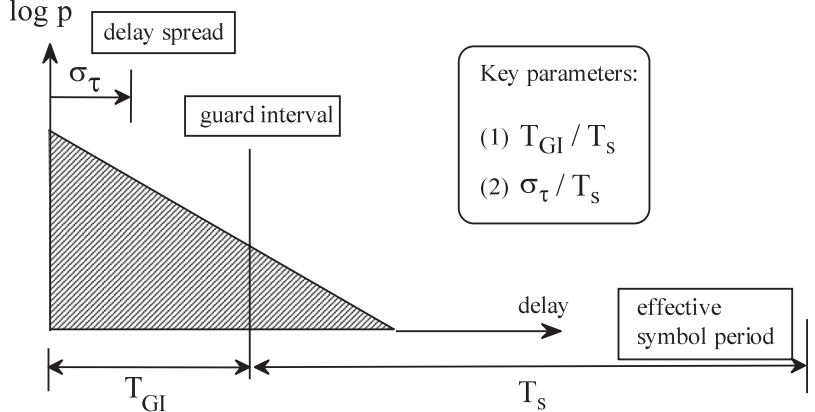

Fig. 14 Key parameters of OFDM performance evaluation in the case of Rayleigh fading with an exponential delay profile [49].

$$
\begin{aligned}
h_{e}(\tau) & =r_{1} \exp \left(j \phi_{1}\right) \delta(\tau)+r_{2} \exp \left(j \phi_{2}\right) \delta\left(\tau-\Delta \tau_{e}\right) \\
& =r_{1} \exp \left(j \phi_{1}\right)\left\{\delta(\tau)+r \exp (j \phi) \delta\left(\tau-\Delta \tau_{e}\right)\right\}
\end{aligned}
$$

where $r$ is the two-path amplitude ratio of $\left(r_{2} / r_{1}\right)$ and $\phi$ is the phase difference $\left(\phi_{2}-\phi_{1}\right)$. By determining $\Delta \tau_{e}$ appropriately, the propagation environment for the evaluation of OFDM transmission characteristic can be expressed as a two-path model where $r_{1}$ and $r_{2}$ show independent Rayleigh distributions. The probability distribution of $\phi$ then becomes a uniform distribution between 0 and $2 \pi$. Delay profile, the tail of which is exceeding the guard interval, causes errors both due to inter-symbol interference (ISI) and inter-carrier interference (ICI) as stated above.

Even if the thermal noise is negligible, BER has a floor value due to ISI and ICI. In this case, $r_{1}$ in Eq. (25) does not contribute to the estimation of the BER floor value so that only $r$ and $\phi$ are key variables. Since $\phi$ is uniformly distributed and does not appear in the equation of the probability distribution as a function, the probability distribution of $r$ should be calculated.

After the statistical manipulation, the joint probability density functions for $r$ and $\phi, f_{p}(r, \phi)$ is finally given by [49],

$$
f_{p}(r, \phi)=\frac{P_{e, 1} P_{e, 2} r}{\pi\left(P_{e, 1} r^{2}+P_{e, 2}\right)^{2}}
$$

For a delay profile of the exponential function type having delay spread $\sigma_{\tau}, f_{p}$ is given by [49]

$$
f_{p}(r, \phi)=\frac{\left\{1-\frac{1}{2} \exp \left(-\frac{T_{\mathrm{GI}}}{\sigma_{\tau}}\right)\right\} \exp \left(-\frac{T_{\mathrm{GI}}}{\sigma_{\tau}}\right) r}{2 \pi\left[\left\{1-\frac{1}{2} \exp \left(-\frac{T_{\mathrm{GI}}}{\sigma_{\tau}}\right)\right\} r^{2}+\frac{1}{2} \exp \left(-\frac{T_{\mathrm{GI}}}{\sigma_{\tau}}\right)\right]^{2}}
$$

The PDF, $f_{p}$, for Nakagami-Rice fading, which is more general expression of multipath fading containing Rayleigh fading as its extreme, was recently presented in Ref. [55].

\subsubsection{BER Floor Estimation and Its Key Parameters}

The BER floor can be expressed in the basic form in Eq. (22) as

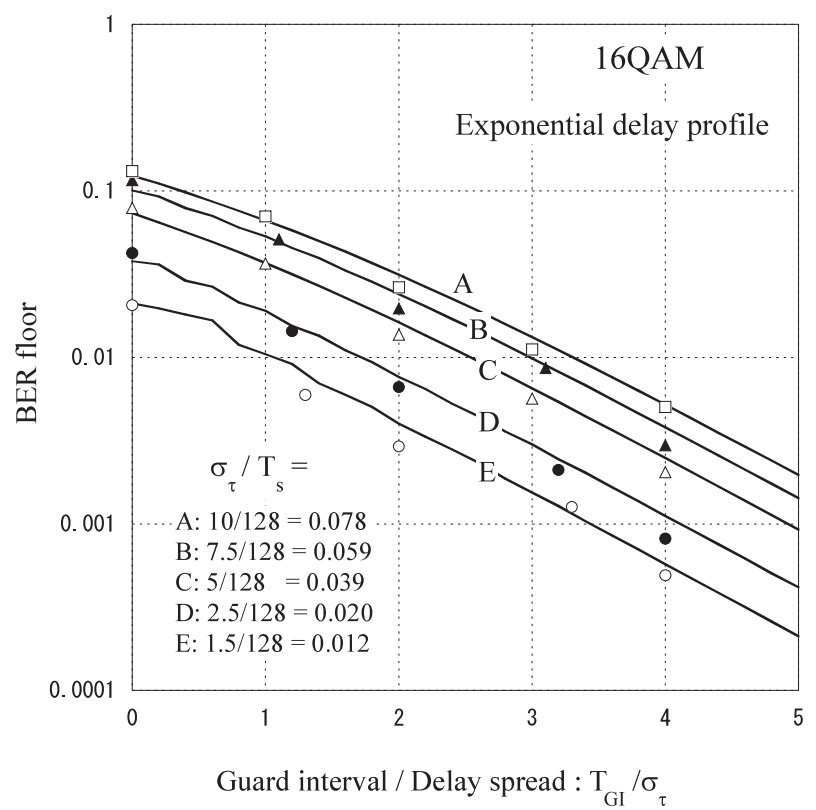

Fig. 15 Comparison of simulated and calculated results of OFDM transmission characteristics for 16 QAM [49].

$$
B E R_{\text {floor }}=\int_{0}^{\infty} \int_{0}^{2 \pi} f_{p}(r, \phi) P_{0}(r, \phi ; \alpha) d \phi d r
$$

where $P_{0}$ is BER of the subject modulation/demodulation scheme for the two-path model as a function of $r, \phi$, and $\alpha$ $\left(=\Delta \tau_{e} / T_{\mathrm{s}}\right)$. Since no simple calculation formula of $P_{0}$ has been established so far, Ref. [49] proposes to calculate $P_{0}$ as a function of $r$ and $\phi$ with sufficiently small step of $\Delta r$ and $\Delta \phi$ in advance and store it as a database. The calculated BER distribution as a function of $r$ and $\phi$ is called "BER map." The BER map is necessary not only for each modulation/demodulation system but also for each $\Delta \tau_{e}$. Therefore, Ref. [56] introduces a method of easily converting a BER map of $\Delta \tau_{e}$ to other values to cope with the differences in $\Delta \tau_{e}$.

From Eq. (27), we have the following two key parameters of the BER floor value if the delay profile is of the exponential function type.

1) Ratio of delay spread $\sigma_{\tau}$ to OFDM effective symbol length $T_{\mathrm{s}}: \sigma_{\tau} / T_{\mathrm{s}}$

2) Ratio of guard interval $T_{\mathrm{GI}}$ to OFDM effective symbol length $T_{\mathrm{s}}: T_{\mathrm{GI}} / T_{\mathrm{s}}$

Either of the two key parameters above may be replaced with the ratio of the delay spread to the guard interval: $\sigma_{\tau} /$ $T_{\mathrm{GI}}$. Figure 14 shows this relationship.

If there exist both irreducible errors $\left(B E R_{\text {floor }}\right)$ and errors due to thermal noise $\left(B E R_{\mathrm{TN}}\right)$, the overall $\mathrm{BER}$ $\left(B E R_{\text {overall }}\right)$ can be approximated to the sum of both errors [52].

$$
B E R_{\text {overall }} \approx B E R_{\text {floor }}\left(\sigma_{\tau}, T_{\mathrm{s}}, T_{\mathrm{GI}}\right)+B E R_{\mathrm{TN}}\left(E_{b} / N_{0}\right)
$$

Figure 15 compares the estimated $B E R_{\text {floor }}$ based on ETP-OFDM model and the results of simulation. The modulation scheme for each subchannel is 16-QAM, the num- 
ber of subchannels is 128, and the delay profile is exponential function type [49]. The good agreement between the results validates the model. In Ref. [49], the transmission characteristics (BER) of the DQPSK and 16-QAM modulation schemes calculated using this model are given for various parameters of OFDM.

References [57], [58] deal with countermeasures against signal deterioration in an environment where the delay spread is beyond the guard interval. The MIMO diversity function is also studied as a method of simultaneously suppressing errors due to thermal noise and inter-symbol interference [59]. These research trends are not within the scope of the survey of propagation models and so are not detailed here.

4.3 Propagation Modeling for OFDM Transmission Characteristic Evaluation under Fast Fading

In OFDM systems, since a serial data stream is split into parallel streams that modulate a group of orthogonal subcarriers, OFDM is generally robust to channel multipath dispersion when the multipath delay does not exceed the guard interval. However, as mentioned in 4.1, the increased symbol duration makes an OFDM system more sensitive to the time variations of mobile propagation channels, namely, the effect of Doppler spreading [60]-[69]. This section introduces the model of Ref. [60] that quantitatively evaluates the relationship between the influence of Doppler spread and the OFDM transmission characteristics based on the works so far.

\subsubsection{Channel Model}

Let us consider a frequency selective randomly varying channel with impulse response $h(t, \tau)$. Within the sufficiently narrow bandwidth of each sub-carrier, compared with the coherence bandwidth of the propagation channel, the sub-channel is modeled as a frequency-flat Rayleigh fading channel. Hence, the channel impulse response $h_{k}(t, \tau)$ for the $k$-th subchannel is denoted as

$$
h_{k}(t, \tau)=a_{k}(t) \sigma(\tau) \quad(k=1,2, \ldots, K)
$$

where the process is a stationary, zero-mean complex Gaussian process. The correlation function is given by

$$
\begin{aligned}
R_{a_{m} a_{n}}(\Delta t) & =\left\langle a_{m}(t+\Delta t) a_{n}^{*}(t)\right\rangle \\
& =R_{1}(\Delta t) R_{2}(m-n)
\end{aligned}
$$

where $R_{1}$ gives the temporal correlation for the process while $R_{2}$ gives the frequency correlation between subcarriers $m$ and $n$. The correlation function given in Eq. (31b) has been frequently used in the literature [60]-[62]. Since we can separate the correlation function to the form given in Eq. (31b), the shape of $R_{2}$ does not affect the following NB analysis. When we assume a Doppler power spectrum modeled as Jakes model [28], namely, the multi-path waves arrive omni-directionally in the azimuth plane, the temporal correlation function is given by

$$
R_{1}(\Delta t)=J_{0}\left(2 \pi f_{D} \Delta t\right)
$$

where $J_{0}$ is the zero-order Bessel function of the first kind.

For most practical multipath channels, the time variation is not very fast that the coherent time is always much larger than the OFDM symbol $T_{\mathrm{s}}$. For such slow fading channels, two-term Taylor series expansion is applicable. The expansion is given by

$$
a_{k}(t)=a_{k}\left(t_{0}\right)+a_{k}^{\prime}\left(t_{0}\right)\left(t-t_{0}\right) \quad t_{0}=T_{\mathrm{s}} / 2,0 \leq t \leq T_{\mathrm{s}}
$$

\subsubsection{Signal to Interference Ratio (CIR)}

In a multipath channel, the received signal is expressed as

$$
\begin{aligned}
r(t)= & h(t, \tau) \otimes s(t)+n(t)=\frac{1}{\sqrt{T_{\mathrm{s}}}} \sum_{k=1}^{K} a_{k}\left(t_{0}\right) s_{k} e^{j 2 \pi f_{k} t} \\
& +\frac{1}{\sqrt{T_{\mathrm{s}}}} \sum_{k=1}^{K} a_{k}^{\prime}\left(t_{0}\right)\left(t-t_{0}\right) s_{k} e^{j 2 \pi f_{k} t}+n(t)
\end{aligned}
$$

where $s_{k}$ is the input transmission signal, and $n(t)$ is the additive white Gaussian noise.

The output of the $i$ th correlator is finally given by,

$$
\hat{d}_{i}=a_{i}\left(t_{0}\right) d_{i}+\frac{T_{\mathrm{s}}}{j 2 \pi} \sum_{\substack{k=1 \\ k \neq i}}^{K} \frac{a_{k}^{\prime}\left(t_{0}\right) d_{k}}{k-i}+n_{i}
$$

where $d_{i}$ and $d_{k}$ are $i$ th and $k$ th input data symbols, and the first term of the right side is the desired signal component, the second term is ICI component and the third is thermal noise.

By considering the correlation function in Eq. (32), the signal to interference ratio (CIR) can be expressed as

$$
C I R=\left\{\frac{\left(T_{\mathrm{s}} f_{\mathrm{D}}\right)^{2}}{2} \sum_{\substack{k=1 \\ k \neq i}}^{K} \frac{1}{(k-i)^{2}}\right\}^{-1}
$$

The CIR curve calculated with (36) is plotted versus the Doppler frequency $f_{\mathrm{D}}$ in Fig. 16 [60]. The OFDM system is assumed to have $K=256$ sub-carriers, with sub-carrier spacing $\Delta f=1 / T_{\mathrm{s}}=7.81 \mathrm{kHz}$ and carrier frequency $f_{\mathrm{c}}=2 \mathrm{GHz}$. The CIR curve given in the figure matches very well with the results given in Refs. [63]-[66]. Including another comparison carried out in Ref. [60], it has been confirmed that the approximation effect expressed by the two-term Taylor series expansion exhibits good accuracy for $f_{\mathrm{D}}$ of larger than $10,000 \mathrm{~Hz}$ where the CIR is less than $-5 \mathrm{~dB}$.

\subsubsection{Digital Transmission Characteristics}

The previous section confirmed that Taylor expansion to the second term would ensure high accuracy in CIR calculations. To calculate the error rate, the CIR probability distribution is essential. In general, the error rate is calculated 


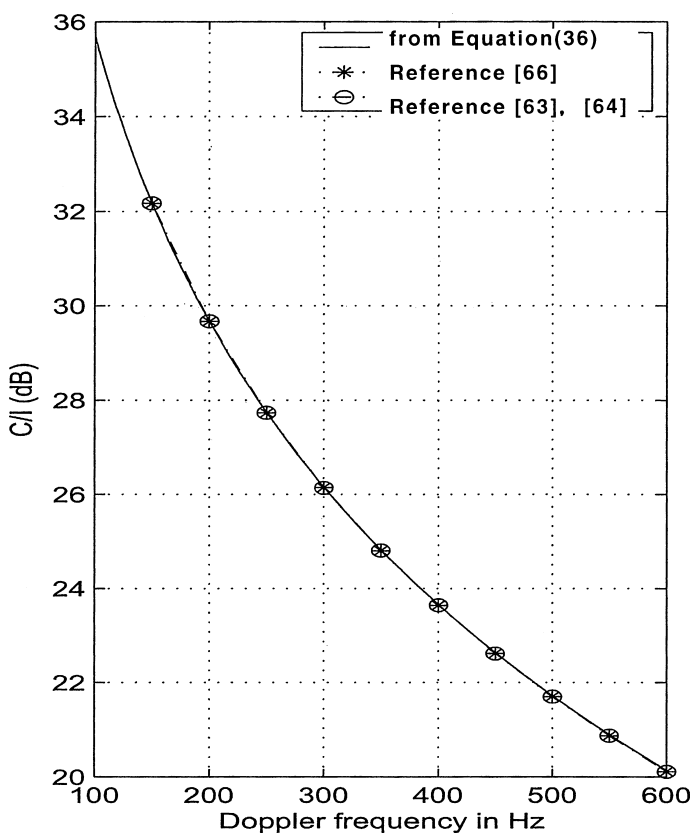

Fig. 16 CIR curbs of an OFDM system, $K=256$ subcarriers, subcarrier distance $\Delta f=7.81 \mathrm{kHz}$, and carrier frequency $f_{\mathrm{c}}=2 \mathrm{GHz}$ [60]. (Wang et al., (C)2006 IEEE)

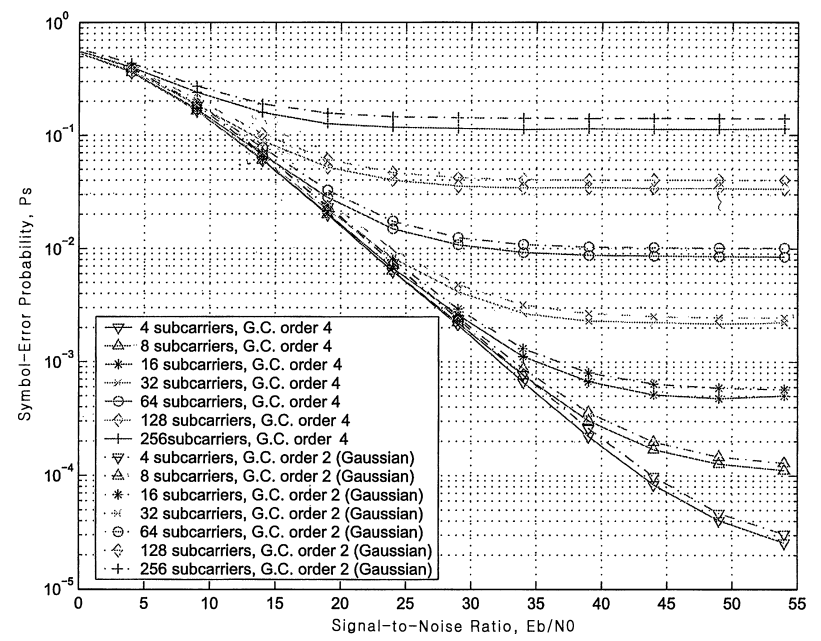

Fig. 17 Performance for 16-QAM OFDM system with $f_{\mathrm{c}}=5 \mathrm{GHz}$, $T_{\mathrm{s}}=1 \mu \mathrm{s}(W=1 \mathrm{MHz})$, and speed of $100 \mathrm{~km} / \mathrm{h}$ [60]. (Wang et al., (C)2006 IEEE)

by regarding interfering waves as additive white Gaussian noise and incorporating them into thermal noise [63]-[69]. References [60], however, clarifies more strictly that the calculated error rate will be closer to the simulated value if the distribution is expressed by a two-dimensional GramCharlier expansion and its coefficient is determined appropriately. Figure 17 shows the comparison of the performance in terms of symbol error probability acquired with the Gaussian approximation (Gram-Charlier expansion of order 2) for the ICI and the non-Gausian approximation (Gram-Charlier expansion of order 4) [60]. The system assumed here is $16-\mathrm{QAM}$ OFDM with $f_{\mathrm{c}}=5 \mathrm{GHz}$, and a speed of $100 \mathrm{~km} / \mathrm{h}$. Symbol period $T_{\mathrm{s}}$ can be obtained by $K T_{0}$ where $T_{0}$ is the symbol period of input signal and assumed to be $T_{0}=1 \mu \mathrm{s}$ here. In the figure, we notice some differences between the strict Gram-Charlier expansion and the general Gaussian approximation for ICI components. However, since the differences are insignificant, the Gaussian approximation is believed to be sufficient for practical purpose.

\section{Propagation Studies for UWB}

\subsection{UWB and Wireless Baseband Transmission (WBT)}

In recent years, UWB has attracted attention as a technology for high-speed transmission as fast as hundreds of Mbps in a personal area network (PAN). The idea of UWB was born from the principle of impulse radio (IR) corresponding to baseband transmission [24], [25], [70]. With the development of high-frequency device technologies, UWB has been studied for low-price, high-speed wireless networks. The great obstacle to the practical use of UWB is the fact that the occupied bandwidth is much greater than that needed by conventional technologies. Authorized by the FCC of the United States [19], UWB research and development are gaining momentum worldwide. In addition to the IR system, multi-band OFDM (MB-OFDM) for transmission through several sub-bands [71] and direct sequence spreadspectrum UWB (DS-UWB) for dual-band transmission [72] have been proposed and are now under review for standardization.

The survey in Ref. [10] relates to UWB antenna and wave propagation. Reference [73] comprehensively introduces UWB applied systems with block diagrams and photos of actual devices, including U.S. patents on UWB radio equipment, tracing the history of UWB technologies. A technical book about UWB has also been published [74] to help understand the technologies.

Wireless baseband transmission (WBT) is also under experimental study as the most advanced UWB transmission scheme that directly radiates and receives continuous baseband signals with an antenna for digital communications [21], [26]. This is an application of baseband wave transmission for cable transmission to wireless transmission. Conventional wireless transmission systems use sinusoidal waves as carriers. In WBT transmission, on the other hand, information is placed on each crest of a wave. In this way, the ultimate wireless transmission system enables ultrahigh-speed transmission. This approach is based on the idea that microwaves will be baseband signals in the era of Gbps or faster wireless communication and can be transmitted to the space without carriers [75].

The next section explains wave propagation channel models and propagation experiment results by focusing on UWB for indoor and outdoor peer-to-peer short-distance communication of 3.1 to $10.6 \mathrm{GHz}$, which is now being standardized, and wireless baseband transmission, research on which has just begun. 


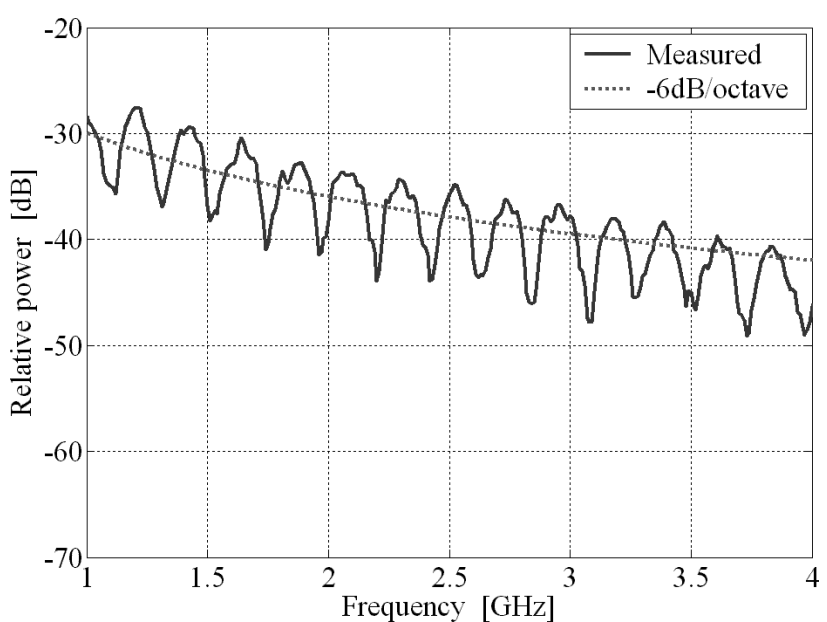

Fig. 18 Overall channel characteristics in two-wave multipath environment where the power gains of Tx and Rx antennas are almost flat in measured frequency range [26].

\subsection{Propagation Channel Model}

As mentioned in Sect. 2.1, a fundamental difference between WB and UWB is whether the frequency characteristics need to be considered when discussing the response in elementary wave transmission. For IR and wireless baseband transmission, the transmission channel characteristics must be expressed in a real-number domain. This is different from NB transmission or WB transmission that uses complex numbers to express channels by conversion into an equivalent low-pass system as mentioned in Sect. 2.2.

Modeling by considering frequency characteristics for elementary waves in multipath propagation was already performed before the development of UWB. References [22], [76] considered variations of frequency characteristics on a single propagation path of reflection, diffraction, and scattering, and analyzed CDMA propagation by considering inband frequency characteristics. By referring [8], [9] and [77], we introduce UWB propagation models.

\subsubsection{Free Space Propagation and Specular Reflection}

By considering the frequency characteristic of antenna gain in transmission and reception and that of free-space propagation loss ( $f^{-2}$ dependence), the direct-wave path power gain $G_{\mathrm{p}}$ can be calculated by the following basic equation:

$$
G_{\mathrm{p}}=G_{\mathrm{TX}}(f) G_{\mathrm{RX}}(f)\left(\frac{c_{0}}{4 \pi d f}\right)^{2}
$$

where $G_{\mathrm{TX}}$ and $G_{\mathrm{RX}}$ are antenna power gains for transmit and receive antennas, $c_{0}$ is the speed of light, $f$ is the radio frequency under consideration, and $d$ is the distance between the antennas. Equation (37) is called Friis' transmission formula. Both antenna gains with no frequency characteristics have resulted in a frequency dependence of $f^{-2}$.

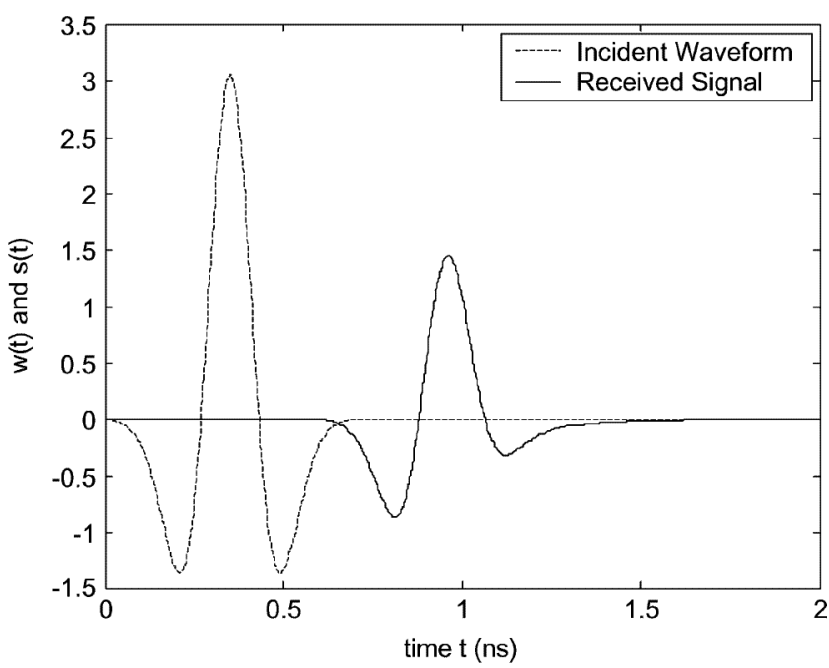

Fig. 19 A UWB pulse diffracted by a half-plane edge [77]. (Qiu, 2004 (C)IEEE)

For reflection, the reflection coefficient of materials encountered in propagation has a frequency characteristic. For commonly used materials, such as ground, asphalt, and concrete wall, the reflection coefficient itself does not have large frequency dependence, excluding special incidence angles such as Brewster's angle against vertical polarized waves. Simple-structured reflection objects do not cause significant waveform distortion. However, if the structure itself is the cause of the frequency characteristic, such as for a layered structure, the influence of the frequency characteristic cannot be ignored [78], [79]. The frequency dependence of building materials is analyzed in detail in Refs. [80], [81].

Figure 18 shows the frequency characteristic of a transmission channel where discone antennas with flat antenna gain at frequencies from 1 to $6 \mathrm{GHz}$ are opposed to each other at one-meter intervals and a metallic plate with specular reflection is placed one meter away from the centerline [26]. The reflection plate is metallic and has no frequency characteristic in this case. However, because of phase interference with direct waves, the combined signals show periodic changes equal to the difference of the path length between the direct and reflected waves. The approximated $f^{-2}$ trend, from Eq. (37), can be seen.

\subsubsection{Diffraction}

The signal intensity against diffraction is generally calculated by using UTD. The diffraction coefficient has a frequency characteristic that should be considered for UWB signals. Several studies have been made considering the frequency characteristic of the diffraction coefficient [9], [76], [77]. Figure 19 compares the waveform of a UWB monopulse signal (second-order Gaussian pulse) received after reflection on a half-plane edge with the original waveform [77]. The edge of the half plane is placed on the direct path so that the peak amplitude becomes half. The waveform is clearly distorted. This indicates the necessity of consider- 
ing the frequency characteristic for diffraction. However, in the case of multipath environments, this kind of frequency dependence might be obscured by other strong frequencydepending factors such as delay spread.

\subsubsection{Rough Surface Scattering}

If a reflection surface has irregularities, rough surface scattering occurs. The scattering is coherent reflection (mirrorlike reflection) if the surface roughness is small while incoherent reflection scattering if it is large. The roughness parameter, $u$, indicating the degree of coherence or incoherence is given by [82], [83]

$$
u(f)=\frac{4 \pi f}{c_{0}} \sigma_{h} \cos \theta_{0}
$$

where $\sigma_{h}$ is the standard deviation of the surface roughness height (rms height), and $\theta_{0}$ is the incident angle measured from the zenith direction to the surface. With $u=1$ as the boundary, the coherent component is larger for $u<1$ and the incoherent component is larger for $u>1$. As we can see from the equation, the roughness parameter is a function of frequency and may need to be considered for UWB. The coherent rough surface reflection component can be expressed as a function of $u$ :

$$
r(u)=r_{0} \exp \left(-u^{2} / 2\right)
$$

where $r_{0}$ is the reflection coefficient for the perfect plane surface when $u=0$.

The frequency characteristic of the incoherent component can be acquired by the theory of scattering. However, statistical techniques are mostly used [84]. For deterministic techniques like ray-tracing, the handling of the incoherent component has not been fully discussed.

\subsubsection{Multipath Environment for UWB}

Actual propagation environments differ from the above single-phenomenon environment and are better represented by a multipath environment composed of several phenomena. In this case, the overall characteristic is evaluated by ray-tracing or from experiments. As expressed by Eq. (4), the frequency characteristics of individual paths should be considered so that they can be combined properly. Since handling the characteristics becomes very complicated, the frequency characteristic as a function of path distance is mainly treated by usual ray-tracing or evaluated using Eq. (3). Therefore, ray-tracing dedicated to UWB has not been much studied. For ray-tracing dedicated to UWB, see Refs. [85] to [87].

In Ref. [88], an indoor environment is measured using waves from 3.1 to $10.6 \mathrm{GHz}$ and the propagation structure is analyzed in detail. The frequency intervals are set to 751 points to achieve a time resolution of $0.13 \mathrm{~ns}$ in a $100 \mathrm{~ns}$ range. In addition, angle-of-departure and angleof-arrival (AOD/AOA) are acquired simultaneously by 700 point space scanning individually at the transmission and

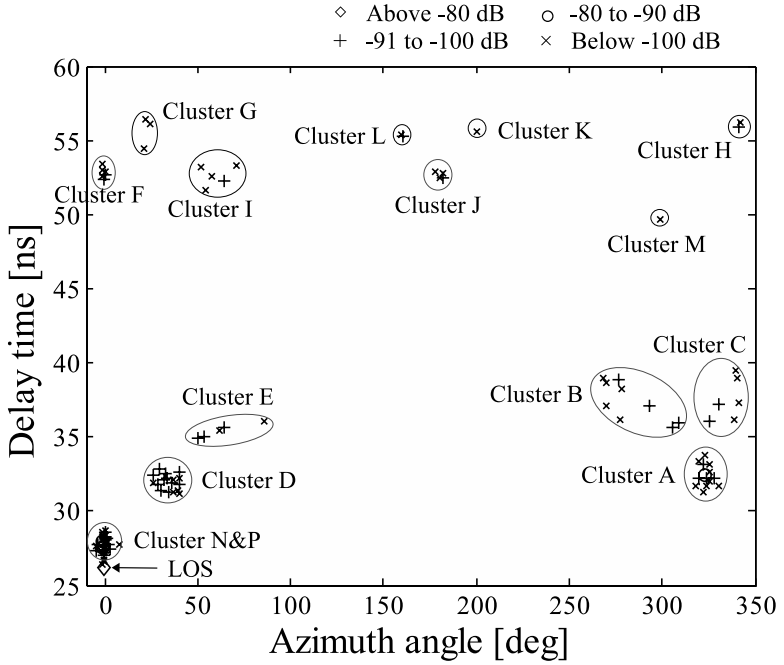

(a) Viewed from the Tx point.

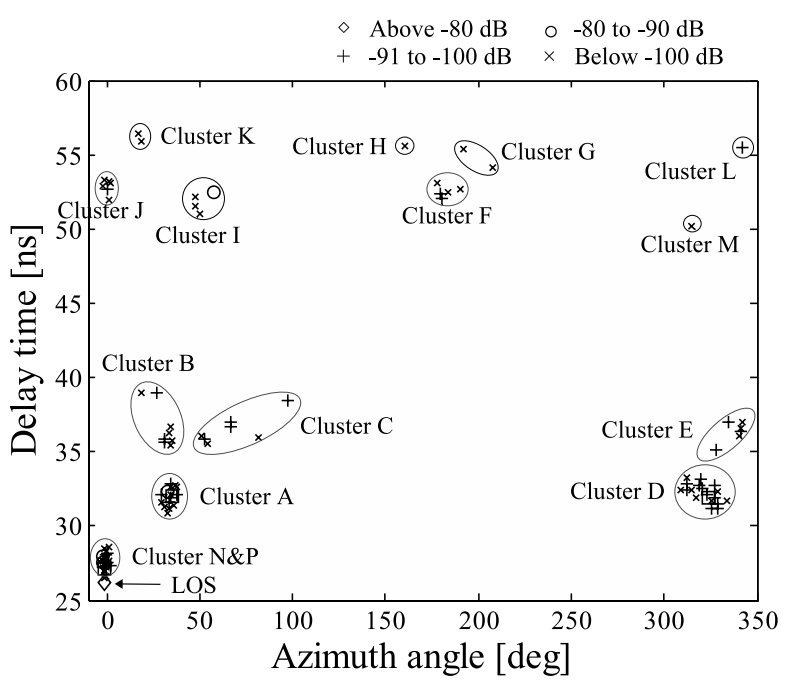

(b) Viewed from the Rx point.

Fig. 20 Clusterization of UWB propagation paths [88].

reception points. Angles were estimated using the SAGE algorithm, which is a super resolution technique based on the maximum likelihood estimation type. The study uses the characteristics of UWB and finds that the reflection objects are grouped into clusters and the delay spread and angular spread are acquired for each cluster. Figure 20 shows a diagram showing the angles and delays of the propagation paths from the view of both transmission and reception points [88]. In the figure, we see that scattered waves arrive together in clusters. With the ray tracing and geometrical consideration, it was reported that clusters $\mathrm{A}, \mathrm{B}, \mathrm{C}, \mathrm{D}, \mathrm{E}$, $\mathrm{F}, \mathrm{J}, \mathrm{N}$, and $\mathrm{P}$ in the figure are single-reflection clusters, and clusters G, H, I, K, L, and M are multiple-reflection clusters. The paper has also reported some useful measured data that are consistent with calculated data by ray tracing. The measurement results clarify that the delay profile of each cluster is of the exponential function type and the angular profile is of the Laplacian distribution as was introduced in Sect. 3.1. The standard deviation of the delay spread of a cluster was 
$0.65 \mathrm{~ns}$ and that of the angular spread was $8.4^{\circ}$. The delay spread value is about $1 / 4$ to $1 / 10$ smaller than other reported results, and the angular spread value is about $1 / 3$ to $1 / 4$ smaller [39], [89], [90]. In future, such detailed analysis of propagation structures will be increasingly important.

\section{Conclusion}

This survey paper has presented propagation studies and models that are expected to contribute to the development of broadband wireless systems. The survey focused on theorybased propagation models, experimental measurement data useful for modeling, and transmission characteristic evaluations using propagation models. The survey did not attempt to cover all papers in the research fields, but rather took several key papers for various relevant subjects and described them in some detail.

As can be seen through this survey, based on continuous and energetic studies so far on radiowave propagation for mobile wireless systems, understanding of propagation phenomena themselves have been successfully advanced and several problems have already been solved. In contrast, however, some systems require the characterization and modeling of multi-dimensional propagation effects mixing with delay characteristic, Doppler characteristic, arrival angle characteristic, and other propagation phenomena, for example, the application of an OFDM system to high-speed mobile terminal communication and introducing adaptive arrays and MIMO. There are growing needs for modeling where propagation characteristics are comprehensively considered in the time, space, and frequency domains. Thus, modeling of compound propagation phenomena will require further study. As for propagation modeling with discriminating WB and UWB more clearly, further study must also be necessary because available data so far are not sufficient in UWB environments.

Greater computing performance is shifting the focus from statistical estimation to the deterministic estimation of propagation represented by ray-tracing. In system-oriented propagation studies, directly useful propagation models are needed for designing new mobile wireless systems, such as the fourth-generation mobile communication, WiMAX, and OFDMA, and for evaluating the transmission characteristics.

\section{References}

[1] J.D. Parsons, The Mobile Radio Propagation Channel, Pentech Press, 1992.

[2] A. Goldsmith, Wireless Communications, Cambridge Univ. Press, 2005

[3] A.F. Molisch, Wireless Communications, IEEE Press, John Wiley \& Sons, 2005

[4] A. Hottinen, O. Tirkkonen, and R. Wichman, Multi-Antenna Transceiver Techniques for $3 \mathrm{G}$ and Beyond, John Wiley \& Sons, 2003.

[5] G. Tsoulos, ed., MIMO System Technology for Wireless Communications, Taylor \& Francis, CRC Press, 2006

[6] R.B. Ertel, P. Cardieri, K.W. Sowery, T.S. Rapaport, and J.F. Reed,
"Overview of spatial channel models for antenna array communication systems," IEEE Pers. Commun., pp.10-22, Feb. 1998.

[7] K. Kalliola, H. Laitinen, P. Vainikainen, M. Toeltsch, J. Laurila, and E. Bonek, "3-D double-directional radio channel characterization for urban macrocellular applications," IEEE Trans. Antennas Propag., vol.51, no.11, pp.3122-3133, 2003.

[8] A.F. Molisch, "Ultrawideband propagation channles-Theory, measurement, and modeling," IEEE Trans. Veh. Technol., vol.54, no.5, pp.1528-1545, 2005.

[9] R.C. Qiu, C. Zhou, and Q. Liu, "Physics-based pulse distortion for ultra-wideband signals," IEEE Trans. Veh. Technol., vol.54, no.5, pp.1546-1555, 2005

[10] T. Maeda, "UWB antennas: Antenna and propagation technologies for ubiquitas ultra high speed wireless communications and perspectives," IEICE Trans. Commun. (Japanese Edition), vol. J88-B, no.9, pp.1586-1600, Sept. 2005

[11] M. Hata, "Empirical formula for propagation loss in land mobile radio sevice," IEEE Trans. Veh. Technol., vol.VT-29, no.3, pp.317$325,1980$.

[12] ITU-R, Propagation data and prediction methods for the planning of short range outdoor radio communication systems and radio local networks in the frequency range $300 \mathrm{MHz}$ to $100 \mathrm{GHz}$, Rec. ITU-R P. 1411-3, 2005.

[13] ITU-R, "Test environments and deployment models," Rec. ITU-R M.1225, Annex 2, pp.194-215, 1997.

[14] J. Forester, et al., "Channel modeling subcommittee final report," IEEE, Doc. IEEE 02490r0P802-15-SG3a, 2003.

[15] M.A. Jensen and J.W. Wallace, "A review of antennas and propagation for MIMO wireless communications," IEEE Trans. Antennas Propag., vol.52, no.11, pp.2810-2824, 2004.

[16] J.P. Kermoal, L. Schumacher, K.I. Pedersen, P.E. Mogensen, and F. Frederiksen, "A stochastic MIMO radio channel model with experimental validation," IEEE J. Sel. Areas Commun., vol.20, no.6, pp.1211-1226, 2002

[17] Y. Karasawa, "MIMO propagation channel modeling," IEICE Trans. Commun., vol.E88-B, no.5, pp.1829-1842, May 2005.

[18] K. Sakaguchi and J. Takada, "Measurement, analysis, and modeling of MIMO propagation channel," IEICE Trans. Commun. (Japanese Edition), vol.J88-B, no.9, pp.1624-1640, Sept. 2005.

[19] FCC, http://www.fcc.gov/Bureaus/Engineering_Technology/Orders/ 2002/fcc02048.pdf

[20] S.A. Hanna, "UWB implications for spectrum management," IEEE Aerosp. Electron. Syst. Mag., vol.19, no.10, pp.17-20, 2004.

[21] J. Kitagawa, T. Taniguchi, and Y. Karasawa, "Wireless baseband transmission experiments," IEICE Trans. Commun., vol.E89-B, no.6, pp.1815-1824, June 2006.

[22] R.C. Qiu, "Wideband wireless multipath channel modeling with path frequency dependence," IEEE Int. Conf. Communications (ICC'96), pp.277-281, Dallas, TX, June 1996.

[23] J.G. Proakis, Digital Communications, Fourth ed., McGraw-Hill, 2001

[24] M.Z. Win and R.A. Scholtz, "Impulse radio: How it works," IEEE Commun. Lett., vol.2, no.2, pp.36-38, 1998.

[25] M.Z. Win and R.A. Scholtz, "Ultra-wide bandwidth time hopping spread spectrum impulse radio for wireless multiple access communications," IEEE Trans. Commun., vol.48, no.4, pp.679-691, 2000.

[26] J. Kitagawa, T. Taniguchi, and Y. Karasawa, "Transmission characteristics of the wireless baseband transmission under multipath environments using real-valued signal analysis," IEICE Trans. Electron. (Japanese Edition), vol.J89-C, no.12, pp.1066-1078, Dec. 2006.

[27] R.H. Clark, "A statistical theory of mobile radio reception," Bell Labs Syst. Tech. J., vol.47, pp.957-1000, July-Aug. 1968.

[28] W.C. Jakes, Microwave Mobile Communications, IEEE Press, 1993. (Reissued)

[29] F. Adachi, M. Feeny, A. Wiliamson, and J. Parsons, "Cross correlation between the envelopes of $900 \mathrm{MHz}$ signals received at a mobile radio base station site,” Proc. IEE, vol.133, pt. F, pp.506-512, 1986 
[30] K.I. Pedersen, P.E. Mogensen, and B.H. Fleury, "Power azimuth spectrum in outdoor environments," Electron. Lett., vol.300, pp.1583-1584, Aug. 1997.

[31] U. Martin, "A directional radio channel model for densely built-up urban areas," Proc. 2nd EPMCC, pp.237-244, Born, Germany, Oct. 1997.

[32] K.I. Pedersen, P.E. Mogensen, and B.H. Fleury, "A stochastic model of the temporal and azimuthal dispersion seen at the base station in outdoor propagation environments," IEEE Trans. Veh. Technol., vol.49, no.2, pp.437-447, 2000.

[33] T. Fujii and H. Omote, "Time-spatial path modeling with multiscattering attenuation factors for wideband mobile propagation," Proc. IEEE Veh. Technol. Conf. (VTC2006 Spring), pp.2656-2660, Melborn, 2006

[34] T. Imai and T. Taga, "Statistical scattering model in urban propagation environment," IEEE Trans. Veh. Technol., vol.55, no.4, pp.1081-1093, 2006.

[35] Y. Karasawa and H. Iwai, "Formulation of spatial correlation statistics in Nakagami-Rice fading environments," IEEE Trans. Antennas Propag., vol.48, no.1, pp.12-18, 2000.

[36] T. Manabe, Y. Miura, and T. Ihara, "Effect of antenna diversity and polarization on indoor multipath propagation characteristics at 60 GHz," IEEE J. Sel. Areas Commun., vol.14, no.2, pp.441-448, 1996

[37] C.P. Lim, J.L. Volakis, K. Sertel, R.W. Kindt, and A.A. Anastasopoulos, "Indoor propagetion models based on rigorous methods for site-specific multipath environments," IEEE Trans. Antennas Propag., vol.54, no.6, pp.1718-1725, 2006.

[38] J. Fuhl, J.P. Rossei, and E. Bonek, "High-resolution 3-D directionof-arrival determination for urban mobile radio," IEEE Trans. Antennas Propag., vol.45, no.4, pp.672-682, 1997.

[39] D.A.M. Saleh and R.A. Valenzuela, "A statistical model for indoor multipath propagation," IEEE J. Sel. Areas Commun., vol.SAC-5, no.2, pp.128-137, 1987.

[40] N. Goto, M. Nakagawa, and K. Itoh, ed., Antennas \& Wireless Communications Handbook, Sec. II-1.3.2 (T. Fujii), Ohmsha, 2006.

[41] T. Fujii, "Delay profile modeling for wideband mobile propagation," Proc. IEEE Veh. Technol. Conf. (VTC2004-Fall), pp.38-42, Los Angels, 2004.

[42] T. Inoue and Y. Karasawa, "Theoretical analysis on the performance of optimal combining for multipath waves distributed in spatial and time domains," IEICE Trans. Commun., vol.E83-B, no.7, pp.14261434, July 2000.

[43] W.C.Y. Lee, Mobile Communications Engineering, McGraw-Hill, 1982.

[44] S. Kozono, "Received signal-level characteristics in a wideband mobile radio channel," IEEE Trans. Veh. Technol., vol.43, no.3, pp.480-486, 1994.

[45] Y. Yamaguchi, K. Suwa, and R. Kawasaki, "Received signal level characteristics for wideband radio channels in line-of-sight microcells," IEICE Trans. Commun., vol.E78-B, no.11, pp.1543-1547, Nov. 1995

[46] T. Inoue and Y. Karasawa, "Theoretical analysis on the level variation properties of wideband signals in the Rayleigh fading environments," PIMRC'99, pp.1263-1267, Osaka, Japan, 1999.

[47] T. Inoue and Y. Karasawa, "Theoretical analysis on level variation of wideband signals in Rayleigh fading environments," IEICE Trans. Commun. (Japanese Edition), vol.J88-B, no.9, pp.1641-1649, Sept. 2005

[48] M. Itami, Well-Understandable OFDM Technologies, Ohmsha, 2005.

[49] Y. Karasawa, N. Gejoh, and T. Izumi, "Modeling and analysis of OFDM transmission characteristics in Rayleigh fading environment in which the delay profile exceeds the guard interval," IEICE Trans. Commun., vol.E88-B, no.7, pp.3020-3027, July 2005.

[50] Y. Karasawa, T. Kuroda, and H. Iwai, "The equivalent transmissionpath model-A tool for analyzing error floor characteristics due to intersymbol interference in Nakagami-Rice fading environments," IEEE Trans. Veh. Technol., vol.46, no.1, pp.194-202, 1997.

[51] H. Iwai and Y. Karasawa, "The theoretical foundation and applications of equivalent transmission-path model for assessing wideband digital transmission characteristics in Nakagami-Rice fading environments," IEICE Trans. Commun., vol.E79-B, no.9, pp.12051214, Sept. 1996.

[52] Y. Karasawa and H. Iwai, "Enhancement of the ETP Model: How to calculate BER due to ISI for wide-band digital transmission in Nakagami-Rice fading environments," IEEE Trans. Veh. Technol., vol.49, no.6, pp.2113-2120, 2000.

[53] Y. Karasawa, "Multipath propagation theory and modeling in wideband mobile radio: The ETP model connecting propagation and systems," The Radio Science Bulletin, no.302, pp.5-15, +p.26, Sept 2002.

[54] N. Gejoh and Y. Karasawa, "OFDM transmission characteristics in multipath environment where the delay spreading exceeds the guard interval analyzed based on the ETP model," IEICE Trans. Commun. (Japanese Edition), vol.J85-B, no.11, pp.1904-1912, Nov. 2002.

[55] Y. Karasawa and C. Vanmany, "OFDM transmission characteristics in multipath environment where the delay spreading exceeds the guard interval analyzed based on the ETP model [V],--In the case of Nakagami-Rice fading environment," IEICE Technical Report, A.P2006-5, 2006

[56] T. Izumi, N. Gejoh, and Y. Karasawa, "Scaling method of BER map for the equivalent transmission-path (ETP) model for OFDM transmission characteristic evaluation," IEICE Trans. Commun. (Japanese Edition), vol.J87-B, no.9, pp.1514-1523, Sept. 2004.

[57] E. Viterbo and K. Fazel, "How to combat long echoes in OFDM transmission schemes: Subband equalization or more powerful channel coding," Proc. GLOBCOM'95, pp.2069-2074, Singapore, Nov. 1995

[58] D. Kim and G.L. Stuber, "Residual ISI cancellation for OFDM with application to HDTV broadcasting," IEEE J. Sel. Areas Commun., vol.16, no.8, pp.1590-1599, 1998.

[59] X. Nguyen, T. Taniguchi, and Y. Karasawa, "Statistical characteristics of MIMO-OFDM transmission systems in multipath environment where the delay spreading exceeds the guard interval," IEICE Trans. Commun. (Japanese Edition), vol.J87-B, no.9, pp.14671476, Sept. 2004

[60] T. Wang, J.G. Proakis, E. Marsy, and J.R. Zeidler, "Performance degradation of OFDM systems due to Doppler spreading," IEEE Trans. Wireless Commun., vol.5, no.6, pp.1422-1432, 2006.

[61] E. Chiavaccini and G.M. Vitetta, "Error performance of OFDM signaling over doubly-selective Rayleigh fading channels," IEEE Commun. Lett., vol.4, no.11, pp.328-330, Nov. 2000.

[62] P. Hoeher, "A statistical discrete-time model for the WSSUS multipath channel," IEEE Trans. Veh. Technol., vol.41, pp.461-468, Nov. 1992

[63] P. Robertson and S. Kaiser, "Analysis of the loss of orthogonality through Doppler spread in OFDM system," Proc. IEEE Globecom, pp.701-706, Dec. 1999.

[64] P. Robertson and S. Kaiser, "The effects of Doppler spread in OFDM (A) Mobile radio systems," Proc. IEEE 50th Veh. Technol. Conf., vol.1, pp.329-333, Sept. 1999.

[65] Y. Li and L.J. Cimini, "Bounds on the interchannel interference of OFDM in time-varying impairments," IEEE Trans. Commun. vol.49, no.3, pp.401-404, March 2001.

[66] Y.S. Choi, P.J. Voltz, and F. Cassara, "On channel estimation and detection for multicarrier signals in fast and frequency selective Rayleigh fading channel," IEEE Trans. Commun., vol.49, no.8, pp.1375-1387, Aug. 2001

[67] M. Russell and G.L. Suber, "Interchannel interference analysis of OFDM in a mobile environment," Proc. IEEE Veh. Technol. Conf., vol.2, pp.820-824, July 1995.

[68] L. Wan and V.K. Dubey, "Bit error probability of OFDM system over frequency nonselective fast Rayleigh fading channels," Elec- 
tron. Lett., vol.36, pp.1306-1307, July 2000.

[69] J. Li and M. Kavehrad, "Effects of time selective multipath fading on OFDM system for broadband mobile applications," IEEE Commun. Lett., vol.3, no.12, pp.332-334, Dec. 1999.

[70] R.A. Scoltz, "Multiple access with time-hoping impulse modulation," Proc. IEEE MILCOM, pp.447-450, 1993.

[71] A. Batra, "Multi-band OFDM physical layer proposal," IEEE P802.15 03/267r2 SG3a, July 2003.

[72] M. Welborn, "XtremeSpectrum CFP presentation," IEEE P802.15 03/153r9 SG3a, July 2003

[73] R.J. Fontana, "Recent system applications of short-pulse ultrawideband (UWB) technology," IEEE Trans. Microw. Theory Tech., vol.52, no.9, pp.2087-2104, 2003.

[74] X. Shen, M. Guizani, R.C. Qiu, and T. Le-Ngoc, Ultra-wideband Wireless Communications and Networks, John Wiley \& Sons, 2006

[75] B. Komiyama, "Introduction to the contents of research at ATR adaptive communications research laboratories," ATR Journal, vol.23, pp.7-10, June 1996.

[76] R.C. Qiu and I.T. Lu, "Multipath resolving with frequency dependence for broadband wireless channel modeling," IEEE Trans. Veh. Technol., vol.48, no.1, pp.273-285, 1999.

[77] R.C. Qiu, "A generalized time domain multipath channel and its application in ultra-wideband (UWB) wireless optimal receiver design: Part II wave-based system analysis," IEEE Trans. Wireless Commun., vol.3, no.11, pp.2312-2324, 2004.

[78] K. Heidary, "Ultra-wideband (UWB) incidence on multiple dielectric interfaces," IEEE Antennas Propag. Society Symp., pp.13151318, 2004.

[79] R. Yao, Z. Chen, and Z. Guo, "An efficient multipath channel model for UWB home networking," IEEE Radio Wireless Conf., pp.511516, 2004.

[80] R. Buehrer, W. Davis, A. Safaai-Jazi, and D. Sweeney, "Ultra wideband propagation measurements and modeling, final report to DARPA NE-TEX program,” Virginia Tech., Tech. Rep., 2004.

[81] A. Muqaibel, A. Safaai-Jazi, A. Bayram, and S. Riad, "UWB through-the-wall propagation and material characteristics," Proc. IEEE Antennas Propag. Society Int. Symp., pp.623-626, 2003.

[82] P. Beckmann and A. Spizzichino, The Scattering of Electromagnetic Waves from Rough Surface, Pergamon Press, 1963.

[83] A. Ishimaru, Wave Propagation and Scattering in Random Media, Academic Press, 1978.

[84] K.W. Lam, Q. Li, L. Tsang, K.L. Lai, and C.H. Chan, "On the analysis of statistical distributions of UWB signal scattering by random rough surfaces based on Monte Carlo simulations of Maxwell equations," IEEE Trans. Antennas Propag., vol.52, no.12, pp.3200-3206, 2004.

[85] H. Sugahara, Y. Watanabe, T. Ono, K. Okanoue, and S. Yamazaki, "Development and experimental evaluations of "RS-2000"-A propagation simulater for UWB systems," Proc. IEEE UWBST 04, pp.76-80, 2004.

[86] B. Uguen, E. Plouhinec, Y. Lostanlen, and G. Chassay, "A deterministic ultra wideband channel modeling," Proc. IEEE UWBST 2002, pp.1-5, 2002.

[87] A.M. Attiya and A. Safaai-Jazi, "Simulation of ultra-wideband indoor propagation," Microw. Opt. Technol. Lett., vol.42, pp.103-107, 2004

[88] H. Tsuchiya, K. Haneda, and J. Takada, "Investigation of ultrawideband propagation channel based on a cluster scheme," IEICE Trans. Fundmentals, vol.E89-A, no.11, pp.3095-3102, Nov. 2006.

[89] Q. Spencer, B. Jeffs, M. Jensen, and A. Swindlehurst, "Modeling the statistical time and angle of arrival characteristics of an indoor multipath channel," IEEE J. Sel. Areas Commun., vol.18, no.3, pp.347360, March 2000.

[90] R.J.-M. Cramer and R.A. Scholtz, "Evaluation of an ultra-wide-band propagation channel," IEEE Trans. Antennas Propag., vol.50, no.5, pp.561-570, 2002.

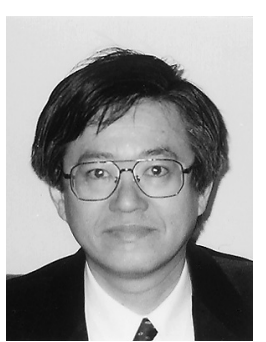

Yoshio Karawasa received B.E. degree from Yamanashi University in 1973 and M.S and Dr. Eng. Degrees from Kyoto University in 1977 and 1992, respectively. He joined KDD R\&D Labs. in 1977. From July 1993 to July 1997, he was a Department Head of ATR Optical and Radio Communications Res. Labs. and ATR Adaptive Communications Res. Labs. both in Kyoto. Currently, he is a professor and the director of the Advanced Wireless Communication research Center (AWCC) in the University of Electro-Communications, Tokyo. Since 1977, he has engaged in studies on wave propagation and antennas, particularly on theoretical analysis and measurements for wave-propagation phenomena, such as multipath fading in mobile radio systems, tropospheric and ionospheric scintillation, and rain attenuation. His recent interests are in frontier regions bridging "wave propagation" and "digital transmission characteristics" in wideband mobile radio systems such as MIMO. Dr. Karasawa received the Young Engineer Award from IECE of Japan in 1983 the Meritorious Award on Radio from the Association of Radio Industries and Businesses (ARIB, Japan) in 1998 , Research Award from ICF in 2006, and two Paper Awards from IEICE in 2006. He is a senior member of the IEEE and a member of SICE of Japan. 